\title{
Association between algal productivity and phycosphere composition in an outdoor Chlorella sorokiniana reactor based on multiple longitudinal analyses
}

Seth Steichen

University of Arizona https://orcid.org/0000-0002-2267-7026

Song Gao

Pacific Northwest National Laboratory

Peter Waller

University of Arizona

Judith Brown ( $\sim$ jbrown@ag.arizona.edu )

https://orcid.org/0000-0003-4669-8443

\section{Research}

Keywords: 16S rRNA gene, benzalkonium chloride, biocide, Health Index, Melainabacteria, microalgal cultivation, microbiome, Vampirovibrionales

Posted Date: January 24th, 2020

DOI: https://doi.org/10.21203/rs.2.21781/v1

License: () (1) This work is licensed under a Creative Commons Attribution 4.0 International License. Read Full License

Version of Record: A version of this preprint was published at Microbial Biotechnology on May 25th, 2020. See the published version at https://doi.org/10.1111/1751-7915.13591. 


\section{Abstract}

\section{Background}

Microalgae as a biofuel source are of great interest. The outdoor Regional Algal Feedstock Testbed (RAFT) was established to optimize algal biomass production in the U.S. sunbelt states. The phycosphere inhabitants, consisting in part of the bacterial community within algal cultures, are hypothesized to contribute to algal productivity. However, little is known about their identity or specific contributions. In this study the bacterial composition of the Chlorella sorokiniana phycosphere was determined over several production cycles in different growing seasons by $16 \mathrm{~S}$ rRNA gene sequencing and identification using the SILVA database.

\section{Results}

The $C$. sorokiniana bacterial phycosphere was sampled during 41 RAFT testbed cycles in two consecutive growing seasons. Eight reactors were sampled twice daily to establish baseline community composition, determined by high-throughput sequencing of 16S ribosomal RNA amplicons. The baseline diversity of the phycosphere increased overall with time during each individual reactor run, based on an increase in Faith's phylogenetic diversity metric versus days post-inoculation $(R=0.66, p<0.001)$. Bacterial assemblages were identified in samples during the stationary, growth, and death stages. During summer months, Vampirovibrio chlorellavorus, an obligate predatory bacterium, was prevalent. The possibility that a sequence correlated with biomass productivity, days post-inoculation, dissolved oxygen, and weather measurements was assessed using a rank-based multinomial regression model. Bacterial sequences assigned to the Rhizobiales, Betaproteobacteriales, and Chitinophagales were positively associated with algal biomass productivity, the industry gold standard for establishing 'efficiency'. Applications of the general biocide, benzalkonium chloride, to a subset of RAFT experiments intended to abate $V$. chlorellavorus appeared to temporarily suppress phycosphere bacterial growth, however, there was no relationship between those bacterial taxa suppressed by benzalkonium chloride and their association with algal productivity, based on multinomial model correlations. Algal health was approximated using a model-based metric, or the 'Health Index' that indicated a robust, positive relationship between $C$. sorokiniana fitness and presence of members belonging to the Burholderiaceae and Allorhizobium-Neorhizobium-Pararhizobium-Rhizobium clade.

\section{Conclusion}

Bacterial community composition was linked to the efficiency of microalgal biomass production and algal health. Thus, bacteria associated positively with algal growth represent potential indicators of culture health and/or as augmentative candidates for enhancing productivity.

\section{Background}

Liquid biofuel has shown great potential to replace a large portion of the global demand for portable energy sources by capturing atmospheric carbon through the process of photosynthesis (Hu et al., 2008). Many green microalgae strains can be readily cultivated for biomass feedstock production. As single-celled organisms they are more efficient than their more complex terrestrial plant relatives (Metting, 1996). While bioethanol crops have been shown to compete with essential food crops by utilizing precious arable land resources (Wigmosta et al., 2011), algae can be grown on marginal lands, achieve high rates of growth, and produce high energy density with some species capable of accumulating as much as $80 \%$ of their total biomass as lipids (Hu et al., 2008; Singh et al., 2011). Large amounts of biomass are required to make microalgal biofuel programs economically feasible, making open outdoor reactors among the most cost effective growth systems, despite certain disadvantages, compared to closed and/or indoor reactors (Jorquera et al., 2010). Open systems also provide excellent platforms for research and discovery because they are exposed to a wide spectrum of abiotic and biotic environmental parameters, of which at least a subset is thought to contribute favorably to algal growth.

Several locations in the southwest region of the United States were strategically selected as United States Department of Energy (DOE) testbed sites to evaluate the economic feasibility of biomass production based on factors such as abundant sunlight, heat, and predicted maximum growth rates (Wigmosta et al., 2011). In previous studies a field isolate of the green microalgae, Chlorella sorokiniana (Shihira and Krauss 1965) designated DOE strain 1412 (Neofotis et al., 2016), exhibited a maximum growth rate of 5.9 day $^{-1}$ at $36^{\circ} \mathrm{C}$, with significant lipid accumulation (Huesemann et al., $2016 \mathrm{a}$ ). As a result, DOE 1412 was prioritized as a primary high temperature test strain for outdoor production, including at the DOE Regional Algal Feedstock Testbed (RAFT) site, The University of Arizona, Tucson, AZ, where temperatures commonly exceed $33^{\circ} \mathrm{C}$ during the summer months. During a three year period from $2015-2018$, the RAFT project conducted cultivation trials under different seasonal, environmental, and operational conditions, including a general biocide treatment to ameliorate the effects of a persistent predatory bacterial pathogen of DOE 1412. (Park et al., 2018; Steichen and Brown, 2018).

Microalgae are known to associate with microorganisms in natural and artificial environments. Prokaryotic bacteria typically represent the most abundant and impactful of these associations (Cole, 1982; Seymour et al., 2017). While axenic cultures of algae are desirable for certain research and industrial applications (Vu et al., 2018), large-scale biomass production systems harbor bacteria naturally, and may encounter bacterial invasions. Previous studies of mass-cultured microalgae recognized that algal-bacterial associations were generally ubiquitous, but considered their contributions to algal growth to be benign or negative (Krauss and Thomas, 1954; Myers et al., 1951). Recent studies have established that complex community dynamics occur between algae and bacteria, and span a range of different ecological relationships (Seymour et al., 2017). Based on these observations, the 'phycosphere concept' has been proposed, as a corollary to the previously recognized 'plant rhizosphere', given robust evidence that bacteria respond chemotactically to algae exudates, thereby defining a zone of algal-bacterial interactions (Bell and Mitchell, 1972). The most commonly detectable phycosphere members are often microbial algal pathogens that cause losses in biomass, which is a quantifiable phenotype. Algae in ecosystems from natural marine environments to biomass production facilities encounter indirect growth inhibition and even direct lysis by amoeba, bacteria, fungi, oomycetes, and viruses (Carney and Lane, 2014; Carney et al., 2014; 
Gachon et al., 2010; Mayali and Azam, 2004; Wang et al., 2010). Production cultures of the DOE 1412 strain of C. sorokiniana used in the studies reported here, and other Chlorella species, have been reported to collapse following attack by the predatory gram-negative cyanobacterium, Vampirovibrio chlorellavorus (Gromov and Mamkaeva) (Ganuza et al., 2016; Park et al., 2018; Soo et al., 2015; Steichen and Brown, 2018-add refs first reports). Alternatively, microbes exist as mutualist symbionts with higher plants, often promoting plant health and contributing to growth and reproduction (Lugtenberg and Kamilova, 2009; Nadeem et al., 2014). Analogous observations have been reported for microalgal species, including C. sorokiniana, which showed significantly higher growth rates in the presence of the diazotrophic, indole-3-acetic acid producing Azospirillum brasilense and Bacilus pumilus bacteria (Amavizca et al., 2017), and certain naturally co-occurring fungi (Watanabe et al., 2005). Recently, microbiome-mediating phenotypes have been shown to account for a portion of the microbiome community (Sze and Schloss, 2018).

Understanding the contributions of individual taxa and networks of interactions with algae within the phycosphere requires the ability to detect the predominant as well as the less abundant types of microbes. Traditional methods of culturing microbes have been shown to identify as few as $1 \%$ of total microbial diversity (Stewart, 2012). The use of culture-independent sequencing of microbial DNA has became a routine technique, relying on conserved regions in the microbial genome. The 16S ribosomal RNA gene is one of the most commonly used molecular markers to distinguish organisms (Woese, 1987) with $~ 1 \%$ sequence divergence indicating different bacterial species (Edgar, 2018). Additional approaches have been developed to facilitate the detection of the most abundant and more rare bacterial species in samples by high-throughput sequencing of the 16S rRNA gene amplicons, making it possible to determine tens of thousands of individual sequence fragments in a sample (Caporaso et al., 2012). Initially, only clustering of sequences representing the different taxa into groups was feasible, based on sequence similarity and referred to as operational taxonomic units (OTUs). However, advancements in computing power and algorithms have permitted the differentiation of individual sequence variants (SVs), enabling the detection of maximum 'signals' within a data set (Callahan et al., 2017; Edgar, 2018).

The parameters recorded and evaluated in this study were $\mathrm{pH}$, algal density, dissolved oxygen, algal growth rate, temperature, precipitation, solar radiation, and wind speed, were measured and archived for each cultivation cycle of C. sorokiniana 1412 in the RAFT reactors. The resultant data were analyzed to identify the most relevant parameters or factors influencing the composition of the C. sorokiniana phycosphere, with an emphasis on the spatial and temporal patterns in the microbiome community over two consecutive growth seasons. The objective was to parse the microbiome community into groups and identify those having the greatest influence on DOE 1412 health, growth, and biomass productivity. Two different sets of experiments were designed and used for determining the composition of bacteria associated with the phycosphere community. The first experimental studies, R41 and R42, were used to conduct replicated, longitudinal analysis of the reactor culture, with twice daily sampling and was used to establish the composition of the 'baseline phycosphere community' under optimal growth conditions. The second set of experiments, R10 through R27 (Table 1), were conducted over two growing seasons in paddlewheel and ARID reactors and were sampled every other day to determine the microbiome community composition based on analysis of $16 \mathrm{~S}$ rRNA gene sequences, for a cross-section of RAFT cultures. The goal of the study was to identify patterns potentially linked to different environmental conditions, including those predicted by modeling to contribute most to algal growth and biomass productivity.

\section{Methods}

\section{Algal culture}

The C. sorokiniana strain DOE1412, previously referred to as NAABB 2412 (Lammers et al., 2017), was isolated from surface water at a collection site in Texas, USA [provided by Dr. J. Polle, Brooklyn College] (Huesemann et al., 2013). The culture was thereafter maintained in the laboratory on BG-11 media containing $17.6 \mathrm{mM}$ of $\mathrm{NaNO}_{3}, 0.22 \mathrm{mM}$ of $\mathrm{K}_{2} \mathrm{HPO}_{4}, 0.03 \mathrm{mM}$ of $\mathrm{MgSO}_{4} \cdot 7 \mathrm{H}_{2} \mathrm{O}, 0.2 \mathrm{mM}$ of $\mathrm{CaCl}_{2} \cdot 2 \mathrm{H}_{2} \mathrm{O}, 0.03 \mathrm{mM}$ of citric acid. $\mathrm{H}_{2} \mathrm{O}, 0.02 \mathrm{mM}$ of ammonium ferric citrate, $0.002 \mathrm{mM}$ of $\mathrm{Na}_{2} \mathrm{EDTA} \cdot 2 \mathrm{H}_{2} \mathrm{O}, 0.18 \mathrm{mM}$ of $\mathrm{Na}_{2} \mathrm{CO}_{3}$, with the addition of trace metals (Rippka and Herdman, 1992). Cultures were maintained by periodic serial transfer on solid BG-11 media containing $30 \mathrm{~g} \cdot \mathrm{L}^{-1}$ of agar. For cultivation, laboratory and outdoor reactor cultures were grown in a modified media designed to obtain high yields while minimizing nutrient inputs, referred to as Pecos media (PE07) (Lammers et al., 2017). The PE07 media contained 1.7 mM of urea $\left(\left(\mathrm{NH}_{2}\right) 2 \mathrm{CO}\right), 0.05 \mathrm{mM}$ of $\mathrm{MgSO}_{4} \cdot 7 \mathrm{H}_{2} \mathrm{O}, 0.3 \mathrm{mM}$ of $\mathrm{NH}_{4} \mathrm{H}_{2} \mathrm{PO}_{4}, 1.4 \mathrm{mM}$ of Potash (KCl), $0.03 \mathrm{mM}$ of FeCl and BG-11 trace metal solution. Field experiments were conducted at the University of Arizona outdoor test site (+32 $\left.16^{\prime} 49.29^{\prime \prime},-110^{\circ} 56^{\prime} 9.82^{\prime \prime}\right)$ in three fresh water reactors, two $762 \mathrm{~L}$ traditional paddlewheel (PW) reactors (Crowe et al., 2012).

\section{Outdoor cultivation and benzalkonium chloride biocide treatment}

Reactors were inoculated with laboratory grown DOE 1412 cells at an optical density of $0.2(\mathrm{OD} 750)$, equivalent of between $3 \cdot 10^{6}$ and $5 \cdot 10^{6}$ cells $\cdot \mathrm{ml}^{-1}$, in PE07 media. Collected biomass was maximized by harvesting $75 \%$ of culture volume during exponential algal growth (OD750 $\geq 1.5$ ). The water and $1 \mathrm{X}$ equivalent media nutrients were immediately replenished. Samples were collected for bacterial phycosphere analysis every other day from selected experiments over a two-year period. Cultures were scored as dying upon observed decrease in algal cell density over two consecutive days. When V. chlorellavorous was considered a risk, the established management practice was the addition of a biocide to abate the attack. To simulate this, one reactor was treated with 2 ppm benzalkonium chloride (BAC) every fourth day, and the other was untreated, and used as the negative control. Biomass productivity was determined based on the ash free dry weight (AFDW) (gms). The AFDW was used to calculate the areal productivity, which was determined by subtracting biomass concentration $\left(\mathrm{g} \cdot \mathrm{L}^{-1}\right)$ from that of the previous measured time point, multiplied by the reactor volume, and divided by the surface area and the number of days from the last AFDW measurement, expressed as $\mathrm{g} \cdot \mathrm{m}^{-2} \cdot$ day $^{-1}$ (Pedroni et al., 2004). A sub-set of replicated experiments were sampled twice daily for bacterial 16S rRNA gene analysis to establish a baseline of typical phycosphere development at the RAFT experiment site. DOE 1412 biomass was cultivated in duplicate raised paddlewheel reactors during two runs treated with BAC (RAFT 41 and 42), and two more runs without the treatment (RAFT 43 and 45). The baseline experiments were run as batches with addition of nutrients at the beginning of the run and no further harvest and water replenishment cycles. 


\section{Measure of culture health}

The health of algal populations has been shown to be partly dependent on its associated phycosphere, both positively and negatively given different compositions. In this study, the difference between the maximum biomass growth rate and the suppressed, observed, biomass growth rate was tested as a measure of culture health. The growth of algae and other phycosphere cells were subject to the constantly varying environmental conditions such as sun light intensity and water temperature. Moreover, the growth slowed down as the increased biomass density induces more self-shading. Therefore, the maximum biomass growth rate of a healthy culture was constantly evolving over time. In this study, a biomass growth model (Huesemann et al., 2016b) was employed to predict the maximum biomass growth rate. The model was built on the maximum specific growth rate as a function of light intensity and water temperature. Self-shading caused light attenuation is calculated based on biomass density. The model was validated by healthy algae pond culture grown under simulated outdoor conditions (Huesemann et al., 2016b). Therefore, it was expected that observed growth rates of healthy cultures could match or exceed those that the model predicted.

In the four outdoor experiments, samples were taken twice daily for AFDW measurements, in the morning and in the evening. The observed growth rate was calculated via,

$$
\mu_{o b s}=\frac{\operatorname{Ln}\left(\frac{A F D W_{e}}{A F D W_{m}}\right)}{t_{e}-t_{m}}
$$

where, $\mu_{\text {obs }}$ is observed growth rate in day ${ }^{-1}, A F D W_{m}$ is the morning AFDW in $g \cdot L^{-1}, A F D W_{e}$ is the evening AFDW in $g \cdot L^{-1}, t_{m}$ is the morning sampling time, and $t_{e}$ is the evening sampling time.

The model simulates the growth during the day by setting the initial AFDW to AFDW $\mathrm{m}$ at morning sampling time. Using the measured solar radiation and water temperature as inputs, by incorporating growth limiting factors (Gao et al., 2018; Khawam et al., 2019), the model yields the AFDW for the evening sampling time, $\mathrm{AFDW}_{\mathrm{e}}$. The modeled growth rate, $\mu_{\text {model }}$, is calculated following Eq. (1) by replacing AFDW with $_{\text {AFDW }}$.

The culture health index $(\mathrm{HI})$ is calculated via,

$$
\text { Health Index }(H I)=-\left|\frac{\mu_{o b s}-\mu_{m o d e l}}{\mu_{o b s}}\right|
$$

Because the model only predicts growth under healthy growth conditions whereas outdoor culture is exposed to various stresses, e.g., contamination, etc., the predicted growth rate is sometimes larger than the observed growth rate $\left(\mu_{\mathrm{obs}}<\mu_{\text {model }}\right)$. To facilitate interpretation and visualization, the negative sign of each $\mathrm{HI}$ value was taken for plotting and regression against log ratios of ASVs such that greater values reflect a higher degree of culture health.

\section{Total DNA isolation}

Total genomic DNA was isolated from algal samples using a modified cetyltrimethylammonium bromide (CTAB) method according to Phillips et al., (2001). Samples were collected from turbulent sections of outdoor reactors into $50 \mathrm{~mL}$ tubes, and the biomass was collected by centrifugation at $4500 \mathrm{xg}$ for $5 \mathrm{~min}$. The supernatant was discarded, and $20 \mathrm{mg}$ of $1.4 \mathrm{~mm}$ stainless steel beads were added to the pellet, with $1 \mathrm{ml}$ CTAB buffer containing $20 \mu \mathrm{L} \beta$ mercaptoethanol. The cells were disrupted using a Mini-Beadbeater-96 (Bio spec. Products Inc., Bartlesville, OK). The supernatant was transferred to a sterile microfuge tube, to which an equal volume of chloroform: isoamyl alcohol (24:1) was added, and the contents was mixed by inverting each tube ten times. The emulsion was broken by centrifugation at $7500 \mathrm{xg}$ for $10 \mathrm{~min}$, and the supernatant was transferred to a microfuge tube. The supernatant was transferred to a sterile microfuge tube and total nucleic acids were precipitated by the addition of $2 / 3$ vol of cold isopropanol. After an overnight ( 16 hr) incubation at $-20^{\circ} \mathrm{C}$, the nucleic acids were collected by microcentrifugation, at $7500 \mathrm{x} \mathrm{g}$ for $10 \mathrm{~min}$. The pellet was washed with $70 \%$ ethanol, air-dried, and dissolved in $20 \mu \mathrm{L}$ TrisHCl buffer (TE), pH 7.2.

\section{S rRNA gene high-throughput sequencing}

The V4 region of the 16S rRNA gene (250 bp) was amplified from the purified DNA preparations by PCR using 515F and 806R primers (Parada et al., 2016), each harboring a barcode such that each product received a unique tag corresponding to the sample (Caporaso et al., 2012) and 5 Prime Hot Mastermix (5 Prime, Germany). Amplicons were quantified using Picogreen (Invitrogen, USA), and $240 \mathrm{ng}$ of PCR amplicons from each sample were pooled. $500 \mu \mathrm{l}$ of pooled library material was run on an electrophoresis gel to remove primer dimers and non-specific PCR amplicons. An $~ 400$ bp band was excised from the gel using the UltraClean DNA Purification and UltraClean PCR Clean-Up Kits (MoBio, USA). The pooled library was analyzed by quantitative PCR amplification using the KAPA Library Quantification Kit (KAPA Biosystems), and 5\% of control PhiX library (Illumina) was added to improve sequencing quality due to the low complexity of 16S rRNA gene amplicon libraries (Kozich et al., 2013). Paired-end sequencing was carried out using 7 pM of the pooled libraries as input on an Illumina MiSeq (Illumina).

\section{S rRNA gene amplicon sequence analysis}

The raw 16S rRNA gene sequence reads were first demultiplexed by assigning each read to the sample of origin according to their unique primer barcode sequences using the 'demux' plugin of QIIME2 (Bolyen et al., 2018) Forward and reverse reads were truncated at 150 bp to remove low quality reads and

Page $4 / 25$ 
Illumina base calling errors were resolved with the DADA2 algorithm as implemented by the 'denoise-paired' method in QIIME2 (Callahan et al., 2016). These steps produced amplicon sequence variants (ASVs) by collapsing high quality reads with $100 \%$ sequence identity into groups, conceptually analogous to operational taxonomic units (OTUs). Each ASV was assigned a taxonomy by a naïve Bayes machine learning algorithm that was trained on the SILVA database (https://www.arb-silva.de/, version 132) implemented in the q2-feature-classifier plugin for QIIME2 (Bokulich et al., 2018). Subsequent analysis of the ASVs was restricted to sequences presumed to represent bacteria associated with the algal cultures, by removing (filtering) those identified as chloroplast or mitochondria sequences. The bacterial ASVs were aligned using the MAFFT algorithm (Katoh and Standley, 2013) and a phylogenetic tree was reconstructed using FastTree 2, with a maximum-likelihood nearest-neighbor interchange calculation (Price et al., 2010). The alpha diversity of the bacterial ASVs identified in each sample was calculated based on frequency, occurrence, and phylogenetic distances using the Faith's Phylogenetic Diversity (PD) metric (Faith, 1992).

\section{Longitudinal analysis of phycosphere development in baseline experiments}

The baseline samples $(n=165)$ were grouped by similarity of membership in the phycosphere using unsupervised clustering and fit to Dirichlet Multinomial Models (DMM) and the ASV frequency per sample as input values. The DMMs were calculated using the Dirichlet Multinomial package for R (Morgan, 2018), using a previously described method (Holmes et al., 2012). The fitness of the model was evaluated by calculating the Laplace approximation on a range of possible Dirichlet components, or number of sample clusters. The samples were ordered by time in culture and assembled into a matrix of DMM cluster membership changes between each day. Transition frequencies were visualized by the adjacency method of the igraph $\mathrm{R}$ library

(https://www.rdocumentation.org/packages/igraph).

\section{Statistical analyses of relationships between phycosphere content and variables over two growing seasons}

A table of ASV frequency for each sample $(n=575)$ and eight variables from the associated metadata file (Additional File 1: Table S1), including benzalkonium chloride treatment (BAC), days after inoculation (DAl), ash free dry weight (AFDW), dissolved oxygen (DO), biomass productivity, temperature, precipitation, solar radiation, and wind speed were used as inputs to build a multinomial regression model based on the Multinomial function in Tensorflow (https://github.com/biocore/songbird/tree/9ed4ede40d8bd8188e93b71d1300c5a1a0a19320) (Morton et al., 2019). The result of the multinomial model was a weighted differential value assigned to each ASV for each of the eight variables. The ASVs with Z-scores greater than 3 (ie; three standard deviations from the mean) were identified as being significantly associated to each of the variables and were summarized by bar plots of their membership to bacterial orders. The relationships between selected bacterial taxa and continuous variables were further corroborated by drawing scatterplots between the log ratios of sequence counts and the variable of interest. Linear relationships were tested by Pearson correlation calculation in base R after testing assumptions for normal distribution and homoscedasticity of residuals.

Pairwise distance metrics were calculated between all samples by the Jaccard, Bray-Curtis, unweighted unifrac, and weighted unifrac methods (Chang et al., 2011; Faith et al., 1987) and principal analyses of the resulting matrices were constructed in QIIME2 (Halko et al., 2011) then visualized using emperor (Vázquez-Baeza et al., 2013). The effect of BAC treatment on bacterial phycospheres was investigated by comparing the ASV content of samples using the PERMANOVA method as implemented in QIIME2 (Anderson, 2001). The ASVs with significantly different relative abundances between untreated and BAC treated samples were identified by conducting analysis of composition of microbiomes (ANCOM) with a centered log ratio transformation (Mandal et al., 2015).

\section{Results}

\section{Baseline community structure analysis}

The typical development of bacterial phycosphere communities in outdoor DOE1412 cultures was established by analyzing the 16S rRNA gene content of samples collected twice-daily from paddlewheel reactors operated under standard practices established during the RAFT project. The growth curves of the biological replicates were similar within runs, particularly during the early exponential growth phase (Fig. 1). Average temperatures of outdoor cultures declined during subsequent experiments while $\mathrm{pH}$ was continuously held near 8 by carbon dioxide injections (Table 1). The diversity of phycosphere bacteria increased with time, post-inoculation, irrespective of benzalkonium chloride treatment $(R=0.66, p<0.001)$ or not $(R=0.47, p<0.001)(F i g .2)$.

Table 1. RAFT baseline experiments continuous data summary 


\begin{tabular}{|lllllll|}
\hline \multicolumn{7}{|c|}{ Culture temperature $\left({ }^{\circ} \mathrm{C}\right)$} \\
\hline Experiment & Average & Average daily maximum & Average daily minimum & pH & EC $(\mathrm{mS} / \mathrm{cm})$ & $\mathrm{DO}(\mathrm{ppm})$ \\
\hline R41PW1 & $25.34 \pm 1.91$ & $32.06 \pm 4.01$ & $20.67 \pm 1.05$ & $7.78 \pm 0.19$ & $-0.74 \pm 1.2$ & $11.78 \pm 6.22$ \\
\hline R41PW2 & $25.07 \pm 1.49$ & $30.47 \pm 2.77$ & $20.9 \pm 1.22$ & $7.9 \pm 0.31$ & $0.67 \pm 0.22$ & $10.76 \pm 6.73$ \\
\hline R42PW1 & $24.38 \pm 1.16$ & $30.38 \pm 2.08$ & $19.78 \pm 1.35$ & $7.87 \pm 0.09$ & $0.43 \pm 0.24$ & $9.12 \pm 4.23$ \\
\hline R42PW2 & $24.11 \pm 1.18$ & $30.45 \pm 2.67$ & $19.83 \pm 1.09$ & $8.04 \pm 0.17$ & $0.93 \pm 0.18$ & $7.36 \pm 4.16$ \\
\hline R43PW1 & $19.64 \pm 2.47$ & $25.24 \pm 3.57$ & $14.37 \pm 1.86$ & $7.83 \pm 0.12$ & $-1.4 \pm 0.6$ & $11.52 \pm 2.94$ \\
\hline R43PW2 & $19.86 \pm 2.66$ & $25.19 \pm 3.56$ & $14.51 \pm 2.02$ & $8.58 \pm 0.23$ & $0.41 \pm 0.39$ & $12.18 \pm 2.26$ \\
\hline R45PW1 & $13.63 \pm 1.95$ & $18.67 \pm 1.98$ & $8.33 \pm 1.1$ & $7.84 \pm 0.08$ & $-2.53 \pm 0.33$ & $9.6 \pm 1$ \\
\hline R45PW2 & $13.46 \pm 1.98$ & $18.6 \pm 1.94$ & $8.45 \pm 2.19$ & $7.98 \pm 0.06$ & $0.74 \pm 0.01$ & $12.09 \pm 1.15$ \\
\hline
\end{tabular}

The phycosphere samples collected during baseline experiments were grouped into six clusters based on the similarity of their ASV frequencies using a Dirichlet multinomial mixture (DMM) modeling approach with lowest Laplace approximation (Additional File 2: Fig. S1) (Holmes et al., 2012). The clusters show a correspondence to the amount of time culture samples were exposed to outdoor cultivation (Fig. 3). Clusters 1 through 4 represented distinct phases of phycosphere development as indicated by samples collected on consecutive days from the same reactor transitioning across these clusters over the course of cultivation (Fig. 3). All the samples in clusters 5 and 6 were collected during experiment R45, which also demonstrated the most distinctly different growth pattern from the other replicates (Fig. 1). The samples in clusters 1 and 2 corresponded with the early algal growth phase, while clusters 3 and 4 consisted of samples collected after day 6 , when the culture algal cell densities began to decline. Thus, the changes in algal growth pattern appeared to be correlated with the composition of bacteria in the phycosphere.

The predominant phyla represented in phycosphere samples were identified as members of the Bacteroidetes and Proteobacteria, comprising $42 \%$ and $40 \%$ of the total 16S rRNA gene sequences respectively. The most abundant ASV was identified as the genus, Vampirovibrio. The most commonly occurring sequence variant among those classified within the Vampirovibrio matched the full length Vampirovibrio chlorellavorus 16 S rRNA gene sequence, at $100 \%$ nucleotide identity, previously determined from the RAFT outdoor reactor samples associated with decline of the C. sorokiniana cultures (Park et al., 2018; Steichen and Brown, 2018). Samples collected during times corresponding to algal culture decline (Fig. 1) were grouped together into DMM clusters 3 and 4 , which were comprised of average relative frequencies of V. chlorellavorus at $46 \%$ and $11 \%$ respectively (Fig. 4). Conversely, clusters 1 and 2 , which corresponded to algal growth phase, were typified by relatively higher proportions of bacteria from the Rheinheimera, Psuedomonas, Massilia, and Bacillus compared to those samples collected from dying cultures.

\section{Bacterial correlations with phycosphere variables during two growing seasons}

Associations between bacterial phycosphere composition and algal culture growth parameters were identified by examining 16S rRNA gene data from samples collected during the baseline experiments together with a broad collection from cultures grown during seasons of optimal daytime growth temperatures $\left(\sim 25-35^{\circ} \mathrm{C}\right)$ for C. sorokiniana DOE 1412 (Additional File 3: Fig. S2). Consistently observed patterns of phycosphere membership across this larger data set represented the most robust interactions that persisted through all the variant environmental conditions that would be encountered during long term cultivation efforts in the Southwestern United States. Bacterial ASV correlations with algal concentration, measured by ash free dry weight (AFDW), showed more variation than correlations with the other seven parameters in a multinomial regression model (Fig. 5). However, AFDW is an instantaneous measurement that is not indicative of algal population growth rate as can be seen when compared to the concentration of oxygen in the media, which is the direct result of active respiration by algae cells. Biomass productivity is a measurement of the change in algal concentration over time, providing a better reflection of cell division or loss as indicated by its close correlation to dissolved oxygen (Additional File 4: Fig. S3). Bacterial ASVs correlated with biomass productivity were assigned predominantly to the orders Rhizobiales, Betaproteobacteriales, and Chitinophagales (Fig. 6). The Rhizobiales and Betaproteobacteriales demonstrated a positive relationship with algal biomass productivity relative to the known C. sorokiniana pathogen, Vampirovibrio chlorellavorus. These relationships were defined primarily by samples collected from declining algal cultures, indicating that a negative log ratio of either of these orders to Vampirovibrionales was consistently associated with algal culture crash (Fig. 8). The frequencies of representatives in the order, Bacillales and order, Pseudomonadales were found to be negatively correlated with days after inoculation of the algal cultures outdoors (Fig. 7), in agreement with the developmental patterns identified by DMM cluster analysis of the baseline experiments (Fig. 4).

Bacterial ASVs assigned to taxa with previously reported relationships to algal growth as well as patterns uncovered during the present study were further investigated by comparing their changing relative abundances across measured biomass productivities in reference to an ASV (6e9594a6005cc8cede0ca7532ff77bfa) assigned to the Sphingobacteriaceae family (Fig. 9). This sequence was selected due to its observed ubiquity in the data set, representing $6.2 \%$ of all sequence reads and being detected in $81.0 \%$ of samples collected. The ASV was also not significantly correlated with biomass productivity by multinomial model analysis, with a differential of -0.06 near the middle of the distribution for the measure (Additional File 5 : Table S2). During the RAFT experiments, the orders Rhodobacteriales and Vampirovibrionales were both negatively correlated with biomass productivity according to multinomial modeling results (Additional File 5: Table S2) and the pattern was further supported by linear regression (Fig. 9b and d). Burkholderiaceae ASV (21c587e98537024a404d6f041c0fb693) and an ASV matching to the previously described algae growth promoting Rhizobium sp. (Kim et al., 2014) were 
selected as examples of possible algal mutualist in the RAFT experiments, but failed to produce significant linear correlations with biomass productivity (Fig. 9a and c).

The most significant relationships between bacterial ASVs and the weather variables; precipitation, solar radiation, and wind speed were generally negative, as indicated by the distributions of outliers in Fig. 5. During the relatively rare periods of precipitation at the study site, the most depleted bacterial orders in the phycosphere samples included Betaproteobacteriales, Chitinophagales, and Sphingomondales that together accounted for 11 of the 22 statistical outliers in the multinomial model (Additional File 5: Table S2). Those statistical outliers of bacteria associated with wind speed were distributed among 18 different taxonomic orders, with the Rhizobiales being the most commonly depleted during high winds, with five ASVs of the order among the statistical outliers. The extreme solar radiation experienced at the test site was linked negatively with a variety of bacterial taxonomies, but most commonly with the orders Betaproteobacteriales, Chitinophagales, and Rhizobiales (Additional File 5: Table S2).

\section{Biocide treatments were consistently associated with changes in bacterial phycosphere composition}

Benzalkonium chloride (BAC) was applied to treat infections of project cultures by Vampirovibrio chlorellavorus bacteria. Applications of BAC reduced accumulation of the pathogen and increased algal growth duration (Steichen and Brown, 2018). The bacterial phycospheres of samples collected from treated cultures shared more similarity to each other than to untreated culture phycospheres in a principal coordinate analysis of their Jaccard beta diversities (Fig. 10a). This observation was supported by a significant difference between the sample groups $(p=0.001)$ when tested by the PERMANOVA method (Fig. 10b) (Table 3). Among the total tested ASVs ( $\mathrm{n}=914), 107$ were determined to be present at significantly different relative abundances between the BAC treated and untreated sample groups based on an ANCOM statistical analysis. The majority of the differentially abundant ASVs shared a negative association with the BAC treatment $(n=65)$ according to their multinomial model differential values, but these bacteria did not show a strong correspondence to biomass productivity (Fig. 10c).

Table 3. Benzalkonium beta group significance PERMANOVA results

\begin{tabular}{|llll|}
\hline Distance metric & pseudo-F & p-value & number of permutations \\
\hline Jaccard & 14.1213 & 0.001 & 999 \\
\hline Bray-Curtis & 15.2898 & 0.001 & 999 \\
\hline Unweighted unifrac & 15.1213 & 0.001 & 999 \\
\hline Weighted unifrac & 10.3279 & 0.001 & 999 \\
\hline$*_{n}=476$ & & & \\
\hline
\end{tabular}

\section{Relationships between bacteria and algal culture health}

During the replicated baseline experiments, algal culture health was quantified by a Health Index (HI) metric, which compared observed growth rates to simulated growth rates calculated using a model that includes minimal bacterial effects. More than $93 \%$ of the observed growth rates were lower than their corresponding simulated growth rate (Additional File 7: Fig. S5a), meaning that greater HI scores approaching zero were indicative of healthy cultures approaching the predicted growth rate. The HI was highest and most stable two to four days following culture inoculation across the replicates (Additional File 7: Fig. S5b) and was most negative during sampling times with low or negative observed growth rates (Additional File 7: Fig. S5c). The negative HI values during the later time points corresponded again with the declining algal cultures during the second half of the baseline experiments. There were fewer overall ASVs significantly correlated with HI (Additional File 8: Fig. S6) than to biomass productivity, but their taxonomic assignments were similar at the order level (Fig. 6). Correlations between selected bacterial ASVs and HI were more significant than either biomass productivity or AFDW (Additional File 9: Fig. S7). Bacterial ASVs from the Burkholderiaceae family and Allorhizobium-Neorhizobium-Pararhizobium-Rhizobium clade were positively correlated with HI with reference to the ubiquitously distributed Hydrogenophaga (Fig. 11a, b), lending further evidence to the possibility of mutualistic promotion of algal growth by these bacteria. The negative correlations of order, Vampirovibrionales and Flavobacterium ASVs to HI demonstrated that the metric identified previously known and putative algal antagonists (Fig. 11c, d).

\section{Discussion}

Culture-independent methods have led to a deeper appreciation of the microbes associated with the human gut (Stewart, 2018), plant rhizosphere (Berendsen et al., 2012), and various open or natural environments (Thompson et al., 2017). The interpretation of 16S rRNA gene sequence data relies on determination of true sequence variants through the use of de-noising algorithms followed by assignment to known taxonomic groupings by comparison with sequences in curated databases (Bokulich et al., 2018). Assemblages of eubacterial and archaeal organisms (Parada et al., 2016) in the phycosphere of experimental, outdoor open algal production reactors (DOE-RAFT project DE-EE0006269, The University of Arizona) were identified by comparative analysis of the 16S rRNA gene sequence. Environmental and experimental variables were collected over more than two years of cultivation and correlated with shifts in the bacterial community structure. The associations between bacteria and microalgal biomass productivity and of culture 'health' under a scenario of repeated rounds of infection of the algal host by the predatory bacterial pathogen, V. chlorellavorous, were investigated to help identify conditions to guide optimization of biomass production in the RAFT reactors. Results demonstrated that the community was dynamic, complex, and responsive to several key environmental 
variables. While the approaches used in this study were able to capture evidence of a fluctuating eubacterial composition associated with the C. sorokiniana algal cultures, the specific mechanisms guiding the observed biological fluxes are not yet known. However, it was possible to apply available information about certain closest bacterial relatives obtained from previously studied phycosphere and rhizosphere systems to develop hypotheses for further testing.

The bacterial phycosphere composition changed throughout the different cultivation cycles and over time, with community diversity increasing (Fig. 2), while certain taxa became more or less prevalent than others (Fig. 3). The fixation of gaseous nitrogen into bioavailable ammonium in exchange for organic carbon is one of the most well-known reactions involving land plants and rhizosphere-associated microorganisms (Berendsen et al., 2012). An analogous phycosphere association has been reported to occur in marine environments where widely-distributed prokaryotes that are associated with their unicellular phytoplankton express the nifH nitrogenase gene, among other dynamic mutualistic contributions (Thompson et al., 2012). The order Rhizobiales, which contains numerous nitrogen-fixing rhizosphere symbionts, was overrepresented among ASVs significantly associated with algal biomass productivity (Fig. 6). Within the Rhizobiales, bacteria belonging to the genus, Devosia, represented two of the ten ASVs whose presence was most closely-associated with C. sorokiniana growth (Table 2). The genus, Devosia contains members that have been previously isolated from root nodules of aquatic leguminous plants (Rivas et al., 2002), suggesting the possibility of a mutualistic partnership with C. sorokiniana involving the partitioning of nitrogen for uptake by algae and other phycosphere members. The Family, Burkholderiaceae sequence variants were very highly correlated with biomass productivity, and contain known nitrogen fixing bacteria (Sawada et al., 2003). The abundance of members of the order, Chitinophagales increased after the initial algal growth phase. This pattern was observed in the baseline experiments for samples collected six-days post-inoculation of reactors (Figs. 3 and 4 ) and Chitinophagales ASVs were positively correlated with number of days in culture during the seasonal monitoring (Fig. 7). The latter group are related to bacteria capable of consuming chitin, and may be opportunists on other phycosphere bacterial species, or the microalgal cell walls that are composed of chitin and chitosan (Blanc et al., 2010) 
Table 2

Most significant sequence variants to biomass productivity according to multinomial model

\begin{tabular}{|c|c|c|c|c|c|c|c|}
\hline Differential & Kingdom & Phylum & Class & Order & Family & Genus & Specie \\
\hline 5.81 & Bacteria & Proteobacteria & Alphaproteobacteria & Rickettsiales & SM2D12 & $\begin{array}{l}\text { unidentified marine } \\
\text { bacterioplankton }\end{array}$ & $\begin{array}{l}\text { unider } \\
\text { marint } \\
\text { bacter }\end{array}$ \\
\hline 5.47 & Bacteria & Proteobacteria & Deltaproteobacteria & Bdellovibrionales & Bdellovibrionaceae & Bdellovibrio & $\begin{array}{l}\text { Bdello } \\
\text { bacter }\end{array}$ \\
\hline 2.86 & Bacteria & Proteobacteria & Gammaproteobacteria & Betaproteobacteriales & Burkholderiaceae & Unassigned & Unass \\
\hline 2.84 & Bacteria & Proteobacteria & Gammaproteobacteria & Betaproteobacteriales & Burkholderiaceae & Unassigned & Unass \\
\hline 2.72 & Bacteria & Bacteroidetes & Bacteroidia & Cytophagales & Cyclobacteriaceae & Algoriphagus & $\begin{array}{l}\text { Algorit } \\
\text { ZHO6? }\end{array}$ \\
\hline 2.72 & Bacteria & Proteobacteria & Alphaproteobacteria & Rhizobiales & Devosiaceae & Devosia & Unass \\
\hline 2.72 & Bacteria & Proteobacteria & Alphaproteobacteria & Rhizobiales & Devosiaceae & Devosia & Unass \\
\hline 2.72 & Bacteria & Proteobacteria & Alphaproteobacteria & Rhizobiales & Xanthobacteraceae & Pseudoxanthobacter & $\begin{array}{l}\text { uncult } \\
\text { proteo }\end{array}$ \\
\hline 2.72 & Bacteria & Bacteroidetes & Bacteroidia & Cytophagales & Spirosomaceae & Unassigned & Unass \\
\hline 2.72 & Bacteria & Proteobacteria & Gammaproteobacteria & Betaproteobacteriales & Burkholderiaceae & Curvibacter & Unass \\
\hline 2.72 & Bacteria & Proteobacteria & Alphaproteobacteria & Rhodobacterales & Rhodobacteraceae & Defluviimonas & Unass \\
\hline 2.72 & Bacteria & Proteobacteria & Alphaproteobacteria & Sphingomonadales & Sphingomonadaceae & Unassigned & Unass \\
\hline 2.72 & Bacteria & Bacteroidetes & Bacteroidia & Chitinophagales & Chitinophagaceae & Flavihumibacter & $\begin{array}{l}\text { uncult } \\
\text { bacter }\end{array}$ \\
\hline 2.72 & Bacteria & Planctomycetes & Planctomycetacia & Pirellulales & Pirellulaceae & Rhodopirellula & Unass \\
\hline 2.72 & Bacteria & Proteobacteria & Gammaproteobacteria & Betaproteobacteriales & Burkholderiaceae & Hydrogenophaga & Unass \\
\hline 2.72 & Unassigned & Unassigned & Unassigned & Unassigned & Unassigned & Unassigned & Unass \\
\hline 2.72 & Bacteria & Proteobacteria & Alphaproteobacteria & Rhizobiales & Hyphomicrobiaceae & Hyphomicrobium & Unass \\
\hline 2.72 & Bacteria & Acidobacteria & $\begin{array}{l}\text { Blastocatellia } \\
\text { (Subgroup 4) }\end{array}$ & Blastocatellales & Blastocatellaceae & uncultured & $\begin{array}{l}\text { uncult } \\
\text { bacter }\end{array}$ \\
\hline 2.72 & Bacteria & Proteobacteria & Alphaproteobacteria & Rhizobiales & Rhizobiaceae & Unassigned & Unass \\
\hline 2.72 & Bacteria & Bacteroidetes & Bacteroidia & Chitinophagales & Chitinophagaceae & Terrimonas & $\begin{array}{l}\text { Terrim } \\
\text { T16R- }\end{array}$ \\
\hline 2.72 & Bacteria & Bacteroidetes & Bacteroidia & Flavobacteriales & Flavobacteriaceae & Flavobacterium & Unass \\
\hline 2.72 & Bacteria & Bacteroidetes & Bacteroidia & Chitinophagales & Chitinophagaceae & Parasegetibacter & $\begin{array}{l}\text { uncult } \\
\text { bacter }\end{array}$ \\
\hline 2.72 & Bacteria & Planctomycetes & Phycisphaerae & Phycisphaerales & Phycisphaeraceae & SM1A02 & metag \\
\hline 2.70 & Bacteria & Proteobacteria & Deltaproteobacteria & Oligoflexales & $0319-6 G 20$ & Unassigned & Unass \\
\hline 2.70 & Bacteria & Proteobacteria & Deltaproteobacteria & Oligoflexales & Oligoflexaceae & uncultured & $\begin{array}{l}\text { uncult } \\
\text { bacter }\end{array}$ \\
\hline 2.69 & Bacteria & Proteobacteria & Gammaproteobacteria & Pseudomonadales & Moraxellaceae & Perlucidibaca & $\begin{array}{l}\text { uncult } \\
\text { bacter }\end{array}$ \\
\hline 2.67 & Bacteria & Proteobacteria & Gammaproteobacteria & Betaproteobacteriales & Burkholderiaceae & Ramlibacter & Unass \\
\hline 2.51 & Bacteria & Proteobacteria & Deltaproteobacteria & Bdellovibrionales & Bdellovibrionaceae & Bdellovibrio & Unass \\
\hline-2.39 & Bacteria & Proteobacteria & Gammaproteobacteria & Pseudomonadales & Pseudomonadaceae & Pseudomonas & Unass \\
\hline-4.65 & Bacteria & Bacteroidetes & Bacteroidia & Cytophagales & Cytophagaceae & Cytophaga & $\begin{array}{l}\text { uncult } \\
\text { bacter }\end{array}$ \\
\hline-5.40 & Bacteria & Verrucomicrobia & Verrucomicrobiae & Verrucomicrobiales & Rubritaleaceae & Luteolibacter & $\begin{array}{l}\text { uncult } \\
\text { bacter }\end{array}$ \\
\hline-7.65 & Bacteria & Proteobacteria & Gammaproteobacteria & Oceanospirillales & Pseudohongiellaceae & Pseudohongiella & metag \\
\hline-8.03 & Bacteria & Patescibacteria & Parcubacteria & $\begin{array}{l}\text { Candidatus } \\
\text { Nomurabacteria }\end{array}$ & Unassigned & Unassigned & Unass \\
\hline-8.36 & Bacteria & Proteobacteria & Alphaproteobacteria & Rhodobacterales & Rhodobacteraceae & Unassigned & Unass \\
\hline
\end{tabular}


Plant growth promoting rhizobacteria (PGPR) have been shown to be phylogenetically diverse, and illicit increased fitness of their terrestrial plant partners by various mechanisms (Berendsen et al., 2012; Lugtenberg and Kamilova, 2009). Several studies have focused on the promotion of algae growth by taxonomically and functionally diverse bacteria. In one example, Rhizobium spp. were the major phycosphere constituents for four different green microalgal species, including Chlamydomonas reinhardtii P.A. Dangeard 1888, Chlorella vulgaris Beyerinck 1890, Scenedesmus sp., and Botryococcus braunii Kützing (Kim et al., 2014). The most commonly occurring full length 16S rRNA gene sequence in the study was identified as a Rhizobium spp. (EU781656) (Kim et al. 2014). In the RAFT reactors, an isolate sharing 100\% nucleotide identity with this Rhizobium spp., ASV (b449f5066387be07ee577a95585d45ca), was present in $39.7 \%$ of samples analyzed during routine monitoring studies. Also, a second Rhizobium spp. (JX255399), following growth in pure culture, was shown to promote the growth of the four algal species following inoculation of the algal cultures (Kim et al. 2014). This bacterial 16S rRNA sequence was 100\% identical to the ASV, 8ce12e88f6b59bb09494567f0d678092, from the RAFT data set for which it was present in 5.6\% of the samples. However, neither the ubiquitous nor the growth-promoting Rhizobium spp. were significantly correlated with biomass productivity, based on coefficients of -0.012 and -0.118 , respectively (Additional File 5: Table S2), and, the linear regression analysis identified one insignificant positive relationship (Fig. 9c). These observations indicate that while phylogenetically-identical bacterial species may apparently occur in different phycospheres, their specific contribution to the community may be specific to habitat and/or algal species composition.

In a recent study, several Azospirillum spp. were shown to increase the growth rate of Chlorella sorokiniana (Shihira \& R.W.Krauss) facilitated by its contribution the hormone, indole-3-acetic acid (IAA) (De-Bashan et al., 2008). Additional studies showed that algae growth increased following inoculation of cultures with Bacillus pumilus (Amavizca et al., 2017; Bashan et al., 2016; Hernandez et al., 2009). The RAFT samples contained seven ASVs that are assigned to the order Azospirillales, however, they were found to be negatively correlated with biomass productivity. While the sole Bacillus sp. ASV identified in the RAFT samples during monitoring had a -0.093 correlation with biomass productivity, two of the six members of the order Bacillales were positively linked to growth. Further, a Chlorella spp. culture having a low growth rate, has been shown to exhibit increased growth when grown in laboratory cultures to which a bacterial consortium from a fast-growing algal culture was used to augment productivity. An analysis of the relative abundances of $16 \mathrm{~S}$ rRNA gene sequences revealed that several Ruegeria sp. were enriched in the fast-growing cultures. While no members of the genus, Ruegeria ASVs were identified among the RAFT samples, the order Rhodobacteriales to which Ruegeria is assigned, was highly correlated with biomass productivity and rapid growth in lab cultures (Richter et al., 2018).

Bacteria that were negatively associated with algal growth during the RAFT project may have been antagonistic or possibly pathogenic to C. sorokiniana, and so are prospective candidates of taxa that could serve as indicators for declining algal culture health. Six ASVs were identified that were significantly negatively associated with C. sorokiniana biomass productivity, based on the multinomial model. These were identified as members of the Rhodobacteraceae, order Nomurabacteria, and the genera Pseudohongiella, Luteolibacter, Cytophaga, and Pseudomonas. (Table 2). Among them, only the genus, Cytophaga has been robustly linked to algicidal activity and killing of diatom species (Imai et al., 1993; Mayali and Azam, 2004), predation on cyanobacteria (Rashidan and Bird, 2001), and accumulation during stationary and death phases of a mixed photobioreactor culture containing members of the family, Scenedesmaceae (Carney et al., 2014).

The predatory cyanobacterium, V. chlorellavorus, is recognized as a virulent pathogen of several species of Chlorella (Coder and Goff, 1986; Ganuza et al., 2016b; Soo et al., 2015). It has been previously shown to be responsible for the death of C. sorokiniana cultures in Arizona at the RAFT site (Steichen and Brown, 2018). Experiments carried out in this study showed that the infection cycle could be managed to some extent through applications of the quaternary ammonium complex, benzalkonium chloride (BAC), a general biocide. However, it is not known whether the apparently marginal negative effects on algae growth were directly related to the biocide itself, or indirectly, because of the potential negative effects it would be expected to have on some or all of the phycosphere bacterial community. In general, there was no significant difference between the biomass productivity of the algal grown in the BAC-treated reactors or in the untreated (negative) control reactors (Additional File 6: Fig. S4a). However, this result bears some uncertainty because during the time in the cultivation season during when reactors were treated with BAC, the outdoor temperatures were higher than several weeks earlier when reactors contained the BAC-untreated cultures (negative control) (Additional File 6: Fig. S4b). An additional caveat is that typically higher temperatures result in faster growth and increased susceptibility of $\mathrm{C}$. sorokiniana to attack by $\mathrm{V}$. chlorellavorus. The hypothesis that algal growth rate was indirectly affected by changes in bacterial community is not supported by the ANCOM results (Fig. 10c) that indicated an overall suppression of the most significantly-affected bacteria, albeit, the latter ASVs did not show a strong relationship to biomass productivity. The distribution of the taxonomic assignments of ASVs significantly affected by the BAC treatment was similar to the overall data set, except for the bacteria assigned to the phyla, Firmicutes, for which only a single ASV was significantly changed by BAC treatment, despite being the third most commonly assigned taxa in the collective data set (Table 4). The Firmicute bacteria, Listeria monocytogenes, encodes an efflux pump that confers resistance to BAC (Kovacevic et al., 2016), offering a mechanism by which members the phylum could have feasibly avoided the potentially toxic effects of the BAC treatment. 
Table 4

Comparison of bacterial phyla significantly changed by benzalkonium chloride treatment to total amplicon sequence variant taxonomic assignments

\begin{tabular}{|c|c|c|c|c|}
\hline Phylum & Total counts & Total proportion (\%) & BAC altered counts & BAC altered proportion (\%) \\
\hline Proteobacteria & 462 & 50.55 & 66 & 61.68 \\
\hline Bacteroidetes & 209 & 22.87 & 22 & 20.56 \\
\hline Firmicutes & 59 & 6.46 & 1 & 0.93 \\
\hline Verrucomicrobia & 48 & 5.25 & 7 & 6.54 \\
\hline Armatimonadetes & 7 & 0.77 & 2 & 1.87 \\
\hline Unassigned & 37 & 4.05 & 2 & 1.87 \\
\hline Chlamydiae & 10 & 1.09 & 2 & 1.87 \\
\hline Planctomycetes & 21 & 2.30 & 2 & 1.87 \\
\hline Cyanobacteria & 11 & 1.20 & 2 & 1.87 \\
\hline Actinobacteria & 16 & 1.75 & 0 & 0.00 \\
\hline Patescibacteria & 11 & 1.20 & 1 & 0.93 \\
\hline Gemmatimonadetes & 5 & 0.55 & 0 & 0.00 \\
\hline Acidobacteria & 4 & 0.44 & 0 & 0.00 \\
\hline BRC1 & 3 & 0.33 & 0 & 0.00 \\
\hline Deinococcus-Thermus & 3 & 0.33 & 0 & 0.00 \\
\hline Dependentiae & 3 & 0.33 & 0 & 0.00 \\
\hline Spirochaetes & 2 & 0.22 & 0 & 0.00 \\
\hline Tenericutes & 2 & 0.22 & 0 & 0.00 \\
\hline Chloroflexi & 1 & 0.11 & 0 & 0.00 \\
\hline
\end{tabular}

Taking quantitative measurements of algal health in outdoor reactor cultures is challenging. On a regular basis, algal cultures are exposed to continuously changing environmental conditions, particularly light intensity and water temperature. Consequently, the growth of the algae and microorganisms that comprise the phycosphere and their interactions are dynamic. Also, algal growth is affected by its own properties and capacity to adapt to and flourish in the collective environment. For example, a 'heathy' dense algal culture would be expected to grow more slowly than a healthy, low-density culture in part owing to increased self-shading. The algal Health Index (HI) computed in this study showed promise for the ability to identify significant bacterial contributors to growth of the target alga. The $\mathrm{HI}$ was found to be more sensitive to identifying bacterial correlations in that there were more significant linear correlations between the pathogen V. chlorellavorus and the putative mutualist bacteria, compared to the biomass productivity and AFDW (Additional File 9: Fig. S7). While the latter two metrics are widely used for assessing industrial biomass production goals, applying the HI calculation developed in this study provided a more accurate depiction of biological fitness of the algal populations analyzed here.

The goal of the RAFT project was to develop a global cultivation system for the efficient production of algal biomass. The microbial members of the phycosphere that were identified in this study and associated with algal health, are possible targets for further assessment. The means by which they influence algal health, whether directly or indirectly, requires further study, including specifically how they function and whether they can be utilized in culture augmentation. Further, some of these taxa may prove to be reliable indicators of productivity for C. sorokiniana and for other algal species as well. In the near term, culture-independent analysis of the entire bacterial community is not well-suited for monitoring, and so the design of more specific assays is needed to enable their easy adaptation for field use. For example, a qPCR assay could be developed to monitor Rhizobiales using the 16S rRNA gene sequence identified in this study as having a tight association with algal growth. These approaches can feasibly provide the ability to obtain rapid, quantitative measures of algal health for the incorporation into models, along with other parameters, that will sound a warning or an alert indicating a need for intervention. With the increasingly economical costs of PCR-amplification and DNA sequencing for routine monitoring and microbiome analysis, the phycosphere community composition can be routinely monitored in reactors and the 'sentinel ASVs' easily identified by specific qPCR amplification. Further, modelling cultures with microbiome analyses coupled to algal Health Index can facilitate more complete assessment of algal culture health than is presently achieved by measuring algal density alone.

\section{Conclusions}

Bacterial community composition was linked to the efficiency of microalgal biomass production and algal health in outdoor reactors that are representative of a practical biofuel operation. The Rhizobia and Chitinophagales bacteria identified here represent positive and negative indicators of $\mathrm{C}$. sorokiniana culture performance respectively. These are candidates for further experimentation to determine whether they can be used to adjust culture performance or monitored for predictions of biomass accumulation or loss. 


\section{Declarations}

Ethics approval and consent to participate

Not applicable

Consent for publication

Not applicable

Availability of data and material

Sequence data have been deposited at the National Center for Biotechnology Information Sequence Read Archive under accession number PRJNA597200.

Competing interests

The authors declare that they have no competing interests.

Funding

Support for this research was provided from the Department of Energy Grant DE-EE0006269.

Authors' contributions

SS designed and carried out the $16 \mathrm{~S}$ amplicon sampling experiments then completed analyses, interpretation, and writing of resulting sequence data. JB designed and provided advise for algal growth and $16 \mathrm{~S}$ amplicon sequencing experiments then assisted in sequence data analyses, interpretation, and manuscript preparation and editing. PW contributed to derivation and implementation of algal health index analyses and helped with first and early drafts of selected manuscript sections, and with subsequent editing of the near final manuscript. SG assisted in derivation and implementation of algal health index, calculated health indices for selected experiments, and contributed to early drafts of relevant sections. All authors read and approved the final manuscript.

\section{Acknowledgements}

The authors would like to express appreciation to Ms. Caitlin. C. Brown for contributions to algal and Vampirovibrio culture maintenance and quality control and experimental design, to Ms. Cassandra Galves and Mr. Stephen Lee for aiding with laboratory experiments, and to the RAFT raceway team. The first author would like to thank Drs. J.K. Brown and K. Ogden for guidance and mentoring throughout this study, a portion of his Ph.D. dissertation. Special thanks to Dr. Daniel Laubitz, Associate Research Scientist, Children's Research Center, College of Medicine for providing expertise and advice in experimental design and downstream analysis of $16 \mathrm{~S}$ rRNA gene sequences, and for conducting the high-throughput sequencing.

\section{CONFLICT OF INTEREST STATEMENT}

The authors declare they have no conflicts of interest.

\section{References}

1. Amavizca, E., Bashan, Y., Ryu, C.-M., Farag, M.A., Bebout, B.M., and de-Bashan, L.E. (2017). Enhanced performance of the microalga Chlorella sorokiniana remotely induced by the plant growth-promoting bacteria Azospirillum brasilense and Bacillus pumilus. Sci. Rep. 7, 41310.

2. Anderson, M.J. (2001). A new method for non-parametric multivariate analysis of variance. Austral Ecol. 26, 32-46.

3. Bashan, Y., Lopez, B.R., Huss, V.A.R., Amavizca, E., and de-Bashan, L.E. (2016). Chlorella sorokiniana (formerly vulgaris) UTEX 2714, a non-thermotolerant microalga useful for biotechnological applications and as a reference strain. J. Appl. Phycol. 28, 113-121.

4. Bell, W., and Mitchell, R. (1972). Chemotactic and growth responses of marine bacteria to algal extracellular products. Biol. Bull. 143, $265-277$.

5. Berendsen, R.L., Pieterse, C.M.J., and Bakker, P.A.H.M. (2012). The rhizosphere microbiome and plant health. Trends Plant Sci. 17, $478-486$.

6. Blanc, G., Duncan, G., Agarkova, I., Borodovsky, M., Gurnon, J., Kuo, A., Lindquist, E., Lucas, S., Pangilinan, J., Polle, J., et al. (2010). The Chlorella variabilis NC64A Genome Reveals Adaptation to Photosymbiosis, Coevolution with Viruses, and Cryptic Sex. Plant Cell 22, $2943-2955$.

7. Bokulich, N.A., Kaehler, B.D., Rideout, J.R., Dillon, M., Bolyen, E., Knight, R., Huttley, G.A., and Gregory Caporaso, J. (2018). Optimizing taxonomic classification of marker-gene amplicon sequences with QIIME 2's q2-feature-classifier plugin. Microbiome 6, 90.

8. Bolyen, E., Rideout, J.R., Dillon, M.R., Bokulich, N.A., Abnet, C., Al-Ghalith, G.A., Alexander, H., Alm, E.J., Arumugam, M., Asnicar, F., et al. (2018). QIIME 2: Reproducible, interactive, scalable, and extensible microbiome data science (PeerJ Inc.).

9. Callahan, B.J., McMurdie, P.J., Rosen, M.J., Han, A.W., Johnson, A.J.A., and Holmes, S.P. (2016). DADA2: High-resolution sample inference from Illumina amplicon data. Nat. Methods 13, 581-583.

10. Callahan, B.J., McMurdie, P.J., and Holmes, S.P. (2017). Exact sequence variants should replace operational taxonomic units in marker-gene data analysis. ISME J. 11, 2639-2643.

11. Caporaso, J.G., Lauber, C.L., Walters, W.A., Berg-Lyons, D., Huntley, J., Fierer, N., Owens, S.M., Betley, J., Fraser, L., Bauer, M., et al. (2012). Ultra-highthroughput microbial community analysis on the Illumina HiSeq and MiSeq platforms. ISME J. 6, 1621-1624.

12. Carney, L.T., and Lane, T.W. (2014). Parasites in algae mass culture. Aquat. Microbiol. 5, 278.

Page $12 / 25$ 
13. Carney, L.T., Reinsch, S.S., Lane, P.D., Solberg, O.D., Jansen, L.S., Williams, K.P., Trent, J.D., and Lane, T.W. (2014). Microbiome analysis of a microalgal mass culture growing in municipal wastewater in a prototype OMEGA photobioreactor. Algal Res. 4, 52-61.

14. Chang, Q., Luan, Y., and Sun, F. (2011). Variance adjusted weighted UniFrac: a powerful beta diversity measure for comparing communities based on phylogeny. BMC Bioinformatics 12, 118.

15. Coder, D.M., and Goff, L.J. (1986). The host range of the chlorellavorous bacterium ("Vampirovibrio chlorellavorus"). J. Phycol. 22, $543-546$.

16. Cole, J.J. (1982). Interactions between bacteria and algae in aquatic ecosystems. Annu. Rev. Ecol. Syst. 13, 291-314.

17. Crowe, B., Attalah, S., Agrawal, S., Waller, P., Ryan, R., Van Wagenen, J., Chavis, A., Kyndt, J., Kacira, M., Ogden, K.L., et al. (2012). A comparison of Nannochloropsis salina growth performance in two outdoor pond designs: conventional raceways versus the arid pond with superior temperature management. Int. J. Chem. Eng. 2012,1-9.

18. De-Bashan, L.E., Antoun, H., and Bashan, Y. (2008). Involvement of indole-3-acetic acid produced by the growth-promoting bacterium Azospirillum spp. in promoting growth of Chlorella vulgaris. J. Phycol. 44, 938-947.

19. Edgar, R.C. (2018). Updating the $97 \%$ identity threshold for 16 S ribosomal RNA OTUs. Bioinformatics 34, 2371-2375.

20. Faith, D.P. (1992). Conservation evaluation and phylogenetic diversity. Biol. Conserv. 61, 1-10.

21. Faith, D.P., Minchin, P.R., and Belbin, L. (1987). Compositional dissimilarity as a robust measure of ecological distance. Vegetatio 69, 57-68.

22. Gachon, C.M.M., Sime-Ngando, T., Strittmatter, M., Chambouvet, A., and Kim, G.H. (2010). Algal diseases: spotlight on a black box. Trends Plant Sci. 15, $633-640$.

23. Ganuza, E., Sellers, C.E., Bennett, B.W., Lyons, E.M., and Carney, L.T. (2016a). A novel treatment protects Chlorella at commercial scale from the predatory bacterium Vampirovibrio chlorellavorus. Microbiol. 7. 848.

24. Gao, S., Waller, P., Khawam, G., Attalah, S., Huesemann, M., and Ogden, K. (2018). Incorporation of salinity, nitrogen, and shading stress factors into the Huesemann algae biomass growth model. Algal Res. 35, 462-470.

25. Halko, N., Martinsson, P., Shkolnisky, Y., and Tygert, M. (2011). An algorithm for the Principal Component Analysis of large data sets. SIAM J. Sci. Comput. $33,2580-2594$.

26. Hernandez, J.-P., de-Bashan, L.E., Rodriguez, D.J., Rodriguez, Y., and Bashan, Y. (2009). Growth promotion of the freshwater microalga Chlorella vulgaris by the nitrogen-fixing, plant growth-promoting bacterium Bacillus pumilus from arid zone soils. Eur. J. Soil Biol. 45, 88-93.

27. Holmes, I., Harris, K., and Quince, C. (2012). Dirichlet multinomial mixtures: generative models for microbial metagenomics. PLOS ONE 7, e30126.

28. Hu, Q., Sommerfeld, M., Jarvis, E., Ghirardi, M., Posewitz, M., Seibert, M., and Darzins, A. (2008). Microalgal triacylglycerols as feedstocks for biofuel production: perspectives and advances. Plant J. 54,621-639.

29. Huesemann, M., Crowe, B., Waller, P., Chavis, A., Hobbs, S., Edmundson, S., and Wigmosta, M. (2016a). A validated model to predict microalgae growth in outdoor pond cultures subjected to fluctuating light intensities and water temperatures. Algal Res. 13, 195-206.

30. Huesemann, M., Crowe, B., Waller, P., Chavis, A., Hobbs, S., Edmundson, S., and Wigmosta, M. (2016b). A validated model to predict microalgae growth in outdoor pond cultures subjected to fluctuating light intensities and water temperatures. Algal Res. 13, 195-206.

31. Huesemann, M.H., Van Wagenen, J., Miller, T., Chavis, A., Hobbs, S., and Crowe, B. (2013). A screening model to predict microalgae biomass growth in photobioreactors and raceway ponds. Biotechnol. Bioeng. 110, 1583-1594.

32. Imai, I., Ishida, Y., and Hata, Y. (1993). Killing of marine phytoplankton by a gliding bacterium Cytophaga sp., isolated from the coastal sea of Japan. Biol. $116,527-532$.

33. Jorquera, O., Kiperstok, A., Sales, E.A., Embiruçu, M., and Ghirardi, M.L. (2010). Comparative energy life-cycle analyses of microalgal biomass production in open ponds and photobioreactors. Bioresour. Technol. 101, 1406-1413.

34. Katoh, K., and Standley, D.M. (2013). MAFFT multiple sequence alignment software version 7: improvements in performance and usability. Mol. Biol. Evol. $30,772-780$.

35. Khawam, G., Waller, P., Gao, S., Edmundson, S., Huesemann, M., Attalah, S., and Ogden, K.L. (2019). Simulation of shading and algal growth in experimental raceways. Algal Res. 41, 101575.

36. Kim, B.-H., Ramanan, R., Cho, D.-H., Oh, H.-M., and Kim, H.-S. (2014). Role of Rhizobium, a plant growth promoting bacterium, in enhancing algal biomass through mutualistic interaction. Biomass Bioenergy 69, 95-105.

37. Kovacevic, J., Ziegler, J., Wałecka-Zacharska, E., Reimer, A., Kitts, D.D., and Gilmour, M.W. (2016). Tolerance of Listeria monocytogenes to quaternary ammonium sanitizers is mediated by a novel efflux pump encoded by emrE. Appl. Environ. Microbiol. 82, 939-953.

38. Kozich, J.J., Westcott, S.L., Baxter, N.T., Highlander, S.K., and Schloss, P.D. (2013). Development of a dual-index sequencing strategy and curation pipeline for analyzing amplicon sequence data on the MiSeq Illumina sequencing platform. Appl. Environ. Microbiol. 79, 5112-5120.

39. Krauss, R.W., and Thomas, W.H. (1954). The growth and inorganic nutrition of Scenedesmus obliquus in mass culture. 123. Plant Physiol. 29, 205-214.

40. Lammers, P.J., Huesemann, M., Boeing, W., Anderson, D.B., Arnold, R.G., Bai, X., Bhole, M., Brhanavan, Y., Brown, L., Brown, J., et al. (2017). Review of the cultivation program within the National Alliance for Advanced Biofuels and Bioproducts. Algal Res. 22, 166-186.

41. Lugtenberg, B., and Kamilova, F. (2009). Plant-Growth-Promoting Rhizobacteria. Annu. Rev. Microbiol. 63, 541-556.

42. Mandal, S., Treuren, W.V., White, R.A., Eggesbø, M., Knight, R., and Peddada, S.D. (2015). Analysis of composition of microbiomes: a novel method for studying microbial composition. Microb. Ecol. Health Dis. 26, 27663.

43. Mayali, X., and Azam, F. (2004). Algicidal bacteria in the sea and their impact on algal Blooms1. J. Eukaryot. Microbiol. 51, $139-144$.

44. Morgan, M. (2018). DirichletMultinomial: Dirichlet-Multinomial Mixture Model Machine Learning for Microbiome Data. 
45. Morton, J.T., Marotz, C., Washburne, A., Silverman, J., Zaramela, L.S., Edlund, A., Zengler, K., and Knight, R. (2019). Establishing microbial composition measurement standards with reference frames. Nat. Commun. 10, 2719.

46. Myers, J., Phillips, J.N., and Graham, J.-R. (1951). On The Mass Culture Of Algae 1. Plant Physiol. 26, 539-548.

47. Nadeem, S.M., Ahmad, M., Zahir, Z.A., Javaid, A., and Ashraf, M. (2014). The role of mycorrhizae and plant growth promoting Rhizobacteria (PGPR) in improving crop productivity under stressful environments. Biotechnol. Adv. 32, 429-448.

48. Neofotis, P., Huang, A., Sury, K., Chang, W., Joseph, F., Gabr, A., Twary, S., Qiu, W., Holguin, O., and Polle, J.E.W. (2016). Characterization and classification of highly productive microalgae strains discovered for biofuel and bioproduct generation. Algal Res. 15, 164-178.

49. Parada, A.E., Needham, D.M., and Fuhrman, J.A. (2016). Every base matters: assessing small subunit rRNA primers for marine microbiomes with mock communities, time series and global field samples. Environ. Microbiol. 18,1403-1414.

50. Park, S.-H., Steichen, S.A., Li, X., Ogden, K., and Brown, J.K. (2018). Association of Vampirovibrio chlorellavorus with decline and death of Chlorella sorokiniana in outdoor reactors. Appl. Phycol.

51. Pedroni, P.M., Lamenti, G., Prosperi, G., Ritorto, L., Scolla, G., Capuano, F., and Valdiserri, M. (2004). EniTecnologie R\&D project on microalgae biofixation of CO2: outdoor comparative tests of biomass productivity using flue gas $\mathrm{CO} 2$ from a NGCC power plant. Proc. GHGT 7, 5-9.

52. Phillips, N., Smith, C.M., and Morden, C.W. (2001). An effective DNA extraction protocol for brown algae. Phycol. Res. 49, 97-102.

53. Price, M.N., Dehal, P.S., and Arkin, A.P. (2010). FastTree 2 - Approximately Maximum-Likelihood Trees for Large Alignments. PLOS ONE 5, e9490.

54. Rashidan, K.K., and Bird, D.F. (2001). Role of predatory bacteria in the termination of a cyanobacterial bloom. Microb. Ecol. 41,97-105.

55. Richter, L.V., Mansfeldt, C.B., Kuan, M.M., Cesare, A.E., Menefee, S.T., Richardson, R.E., and Ahner, B.A. (2018). Altered Microbiome Leads to Significant Phenotypic and Transcriptomic Differences in a Lipid Accumulating Chlorophyte. Environ. Sci. Technol. 52, 6854-6863.

56. Rippka, R., and Herdman, M. (1992). Pasteur culture collection of cyanobacterial strains in axenic culture. Cat. Taxon. Handb. 1, 103.

57. Rivas, R., Velázquez, E., Willems, A., Vizcaíno, N., Subba-Rao, N.S., Mateos, P.F., Gillis, M., Dazzo, F.B., and Martínez-Molina, E. (2002). A New Species of Devosia That Forms a Unique Nitrogen-Fixing Root-Nodule Symbiosis with the Aquatic Legume Neptunia natans (L.f.) Druce. Appl Env. Microbiol 68, 5217-5222.

58. Sawada, H., Kuykendall, L.D., and Young, J.M. (2003). Changing concepts in the systematics of bacterial nitrogen-fixing legume symbionts. J. Gen. Appl. Microbiol. 49, 155-179.

59. Seymour, J.R., Amin, S.A., Raina, J.-B., and Stocker, R. (2017). Zooming in on the phycosphere: the ecological interface for phytoplankton-bacteria relationships. Nat. Microbiol. 2, 17065 .

60. Singh, A., Nigam, P.S., and Murphy, J.D. (2011). Renewable fuels from algae: An answer to debatable land based fuels. Bioresour. Technol. $102,10-16$.

61. Soo, R.M., Woodcroft, B.J., Parks, D.H., Tyson, G.W., and Hugenholtz, P. (2015). Back from the dead; the curious tale of the predatory cyanobacterium Vampirovibrio chlorellavorus. Peer J 3, e968.

62. Steichen, S.A., and Brown, J.K. (2018). Real-time quantitative detection of Vampirovibrio chlorellavorus, an obligate bacterial pathogen of Chlorella sorokiniana. J. Appl. Phycol.

63. Stewart, C. (2018). Temporal Development of the Gut Microbiome in Early Childhood: The TEDDY Study StewartLab/Stewart_TEDDY_Microbiome_Analysis.

64. Stewart, E.J. (2012). Growing Unculturable Bacteria. J. Bacteriol. 194, 4151-4160.

65. Sze, M.A., and Schloss, P.D. (2018). Leveraging Existing 16S rRNA Gene Surveys To Identify Reproducible Biomarkers in Individuals with Colorectal Tumors. MBio 9.

66. Thompson, A.W., Foster, R.A., Krupke, A., Carter, B.J., Musat, N., Vaulot, D., Kuypers, M.M.M., and Zehr, J.P. (2012). Unicellular Cyanobacterium Symbiotic with a Single-Celled Eukaryotic Alga. Science 337, 1546-1550.

67. Thompson, L.R., Sanders, J.G., McDonald, D., Amir, A., Ladau, J., Locey, K.J., Prill, R.J., Tripathi, A., Gibbons, S.M., Ackermann, G., et al. (2017). A communal catalogue reveals Earth's multiscale microbial diversity. Nature 551, 457-463.

68. Vázquez-Baeza, Y., Pirrung, M., Gonzalez, A., and Knight, R. (2013). EMPeror: a tool for visualizing high-throughput microbial community data. GigaScience 2, 16.

69. Vu, C.H.T., Lee, H.-G., Chang, Y.K., and Oh, H.-M. (2018). Axenic cultures for microalgal biotechnology: Establishment, assessment, maintenance, and applications. Biotechnol. Adv. 36, 380-396.

70. Wang, X., Li, Z., Su, J., Tian, Y., Ning, X., Hong, H., and Zheng, T. (2010). Lysis of a red-tide causing alga, Alexandrium tamarense, caused by bacteria from its phycosphere. Biol. Control 52,123-130.

71. Watanabe, K., Takihana, N., Aoyagi, H., Hanada, S., Watanabe, Y., Ohmura, N., Saiki, H., and Tanaka, H. (2005). Symbiotic association in Chlorella FEMS Microbiol. Ecol. 51, 187-196.

72. Wigmosta, M.S., Coleman, A.M., Skaggs, R.J., Huesemann, M.H., and Lane, L.J. (2011). National microalgae biofuel production potential and resource demand. Water Resour. Res. 47, W0OH04.

73. Woese, C.R. (1987). Bacterial evolution. Microbiol. Rev. 51, 221.

\section{Additional Files}


$. x \mid s x$ file

Table S1. RAFT 16S rRNA gene sample metadata table.

Additional File 2:

.pdf file

Figure S1. Optimal Dirichlet Multinomial Model cluster number for baseline data set. Goodness of fit measured by Laplace approximation as a function of the number of Dirichlet components. Lower Laplace values indicate increased model fitness to the data set.

\section{Additional File 3:}

.png file

Figure S2. Monthly distribution of survey data set sample collections.

\section{Additional File 4:}

.png file

Figure S3. Comparisons of algal growth metrics. Scatter plots of ash free dry weight (AFDW), biomass productivity (biomass_prod), daily average dissolved oxygen (DO), and days after inoculation (DAl) were drawn based on measurements taken during phycosphere sampling time points. Lines represent Pearson correlation with shaded areas indicating the $95 \%$ confidence intervals.

\section{Additional File 5:}

.$x I s x$ file

Table S2. Multinomial model differentials for all amplicon sequence variants and their taxonomic assignments

\section{Additional File 6:}

.png file

Figure S4. Distributions of A) biomass productivity $(p=0.51)$ and B) temperature $(p=2.32 e-10)$ measurements for samples collected from cultures treated (blue) or untreated (orange) with benzalkonium chloride.

\section{Additional File 7:}

.png file

Figure S5. Comparisons of simulated and observed algal growth rates during RAFT baseline experiments. Observed and simulated algal culture growth rates for all four runs are shown (a) with correlation curves drawn based on loess approximation and $95 \%$ confidence intervals indicated by shaded areas. Boxplots (b) indicating the distributions of calculated absolute relative error of growth rates $(\mathrm{mu})$ are displayed by days after inoculation, and the relationship between observed growth rate and calculated absolute relative error of growth rate (c) for the four duplicate runs.

\section{Additional File 8:}

\section{.png file}

Figure S6. Bacterial orders most highly correlated with algal Health Index (HI). Counts of sequence variants with multinomial regression differential values for days after inoculation that have a z score greater than three. The sequence variants are grouped by their assigned taxonomies at the order level, and bars are color coded by the direction of the correlation.

\section{Additional File 9:}

\section{.png file}

Figure S7. Comparisons of putative algal culture performance metrics for identification of bacterial interactions. The log ratios of the relative abundance of ac) Allorhizobium-Neorhizobium-Pararhizobium-Rhizobium (ANPR) and d-f) Vampirovibrio sp. to Methylophilus for each sample are plotted by their corresponding algal $\mathrm{HI}$ measure, biomass productivity, and ash free dry weight (AFDW). Lines and grey areas represent linear regression and $95 \%$ confidence intervals respectively, along with the $\mathrm{R}$ fitness and $\mathrm{p}$ significance values based on a Pearson correlation test.

\section{Figures}




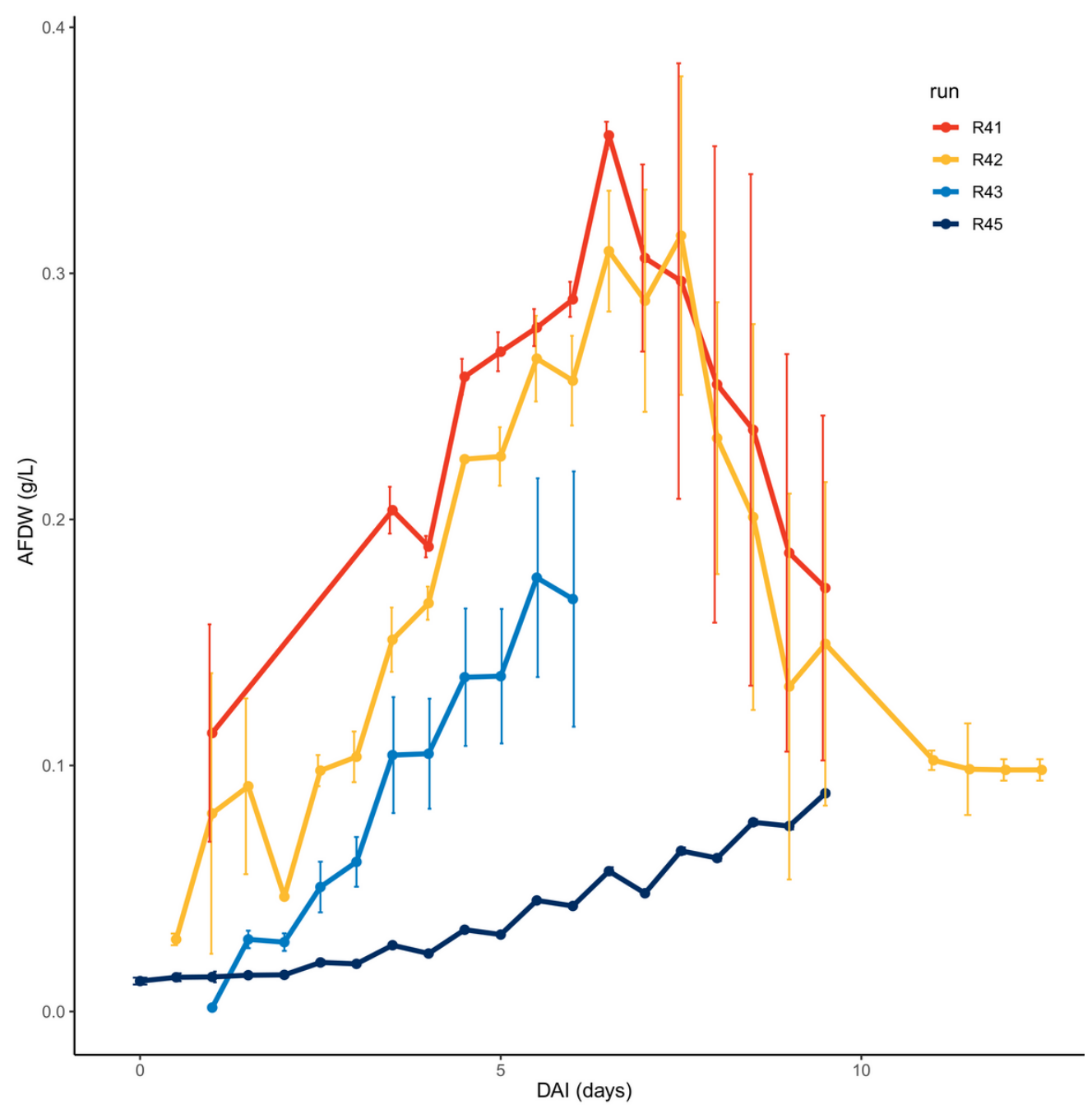

Figure 1

Growth of RAFT baseline experiment cultures. Each line depicts the mean ash free dry weight $(\mathrm{g} / \mathrm{L})$ concentrations for each duplicate run. Error bars represent standard deviation ( $n=2)$. Benzalkonium chloride treatments were applied to R41 and R42 cultures. 


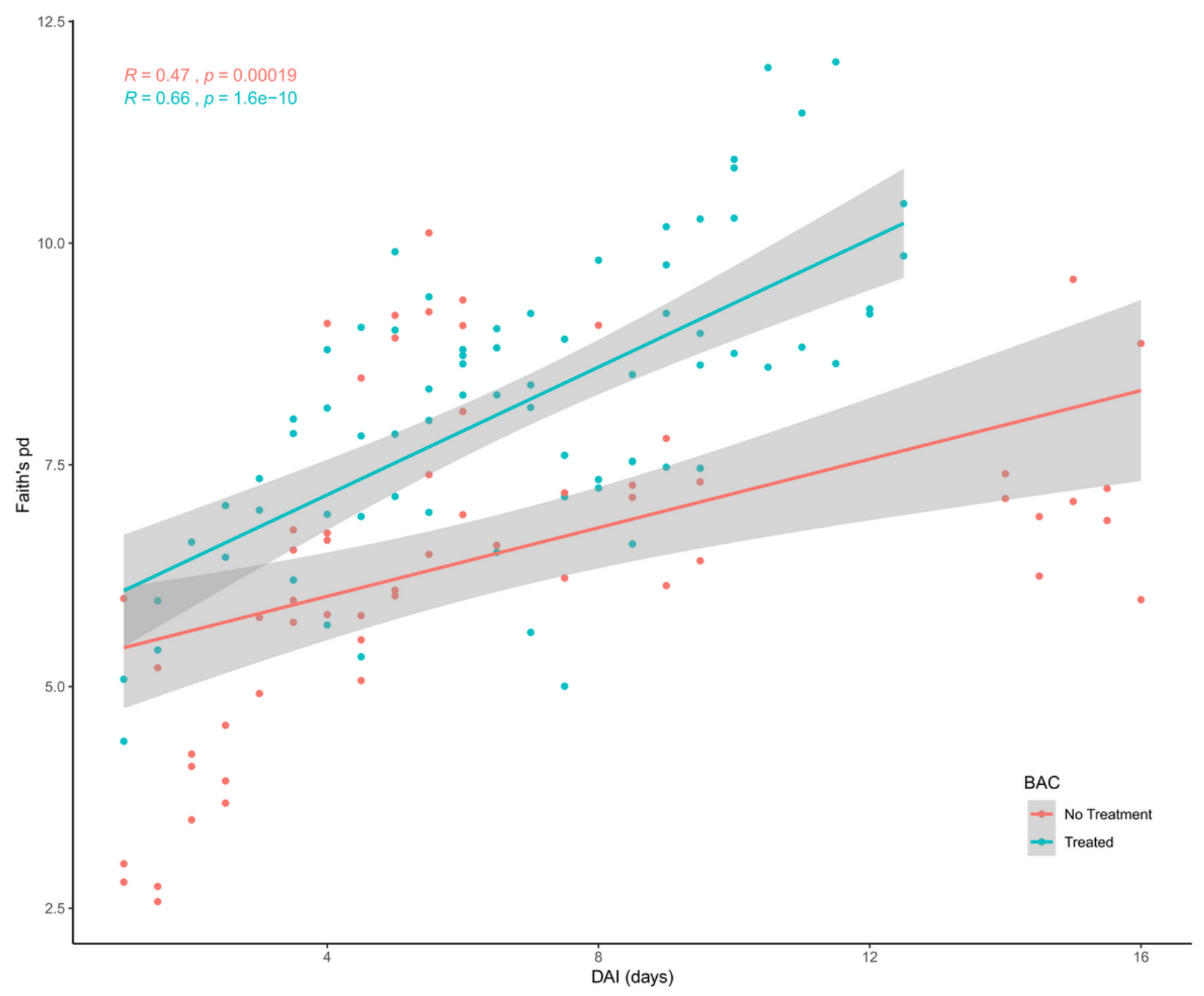

Figure 2

Baseline phycosphere diversity is positively correlated with duration in outdoor reactors. 16S rRNA gene sequence diversity data plotted by days after inoculation in outdoor reactor. Lines indicate linear regression with 95\% confidence intervals in grey. The R fit values and $\mathrm{p}$ values were determined by Pearson correlation analyses. 


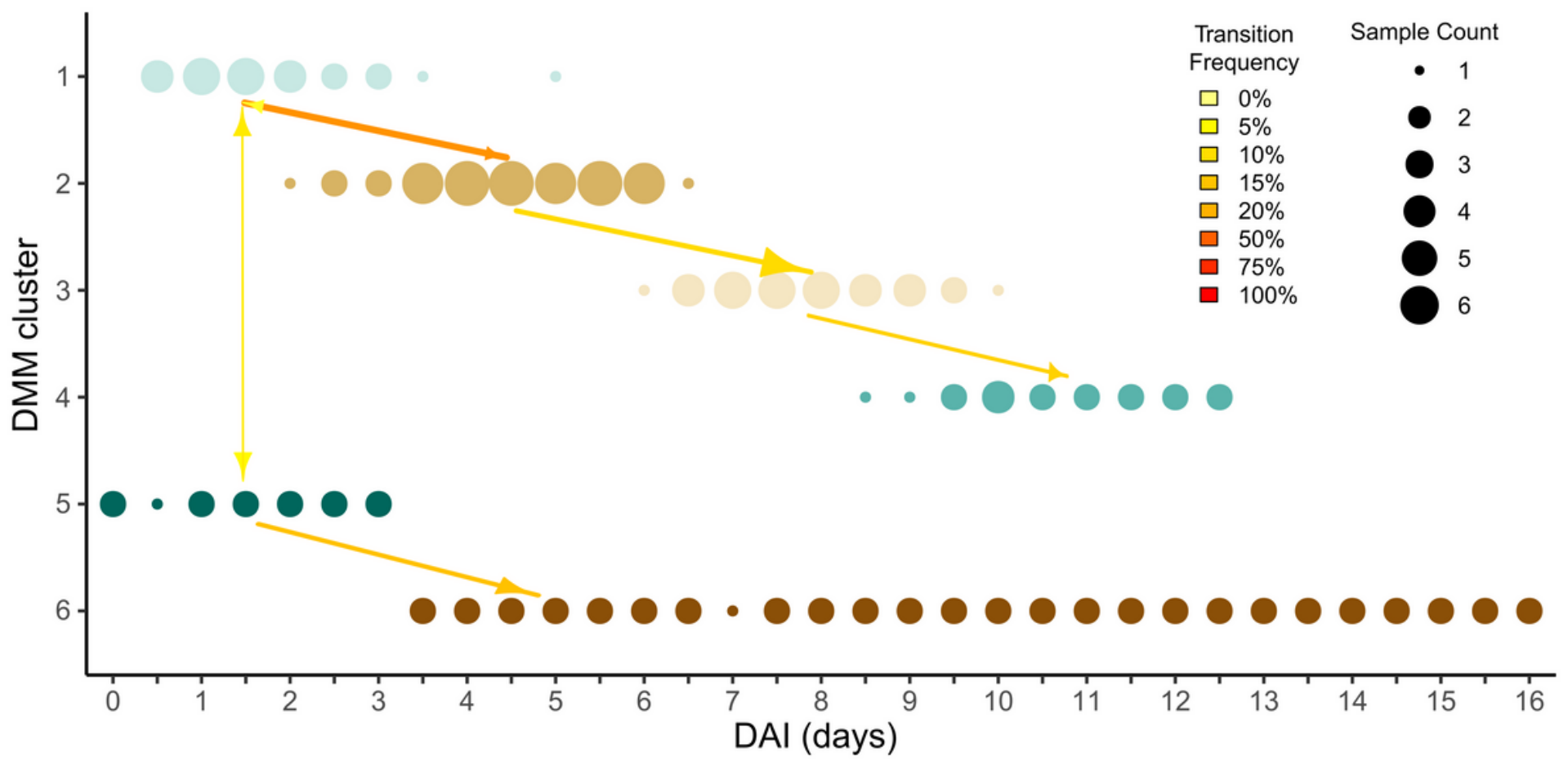

Figure 3

DMM clusters group together based on cultivation time in the outdoor RAFT reactor. Circles are colored by their DMM cluster assignment and sizes are scaled by the number of samples collected at the same time point for the same DMM cluster. Number of samples collected from each of the same reactors on the subsequent day, are indicated by vector lines between the DMM clusters. 


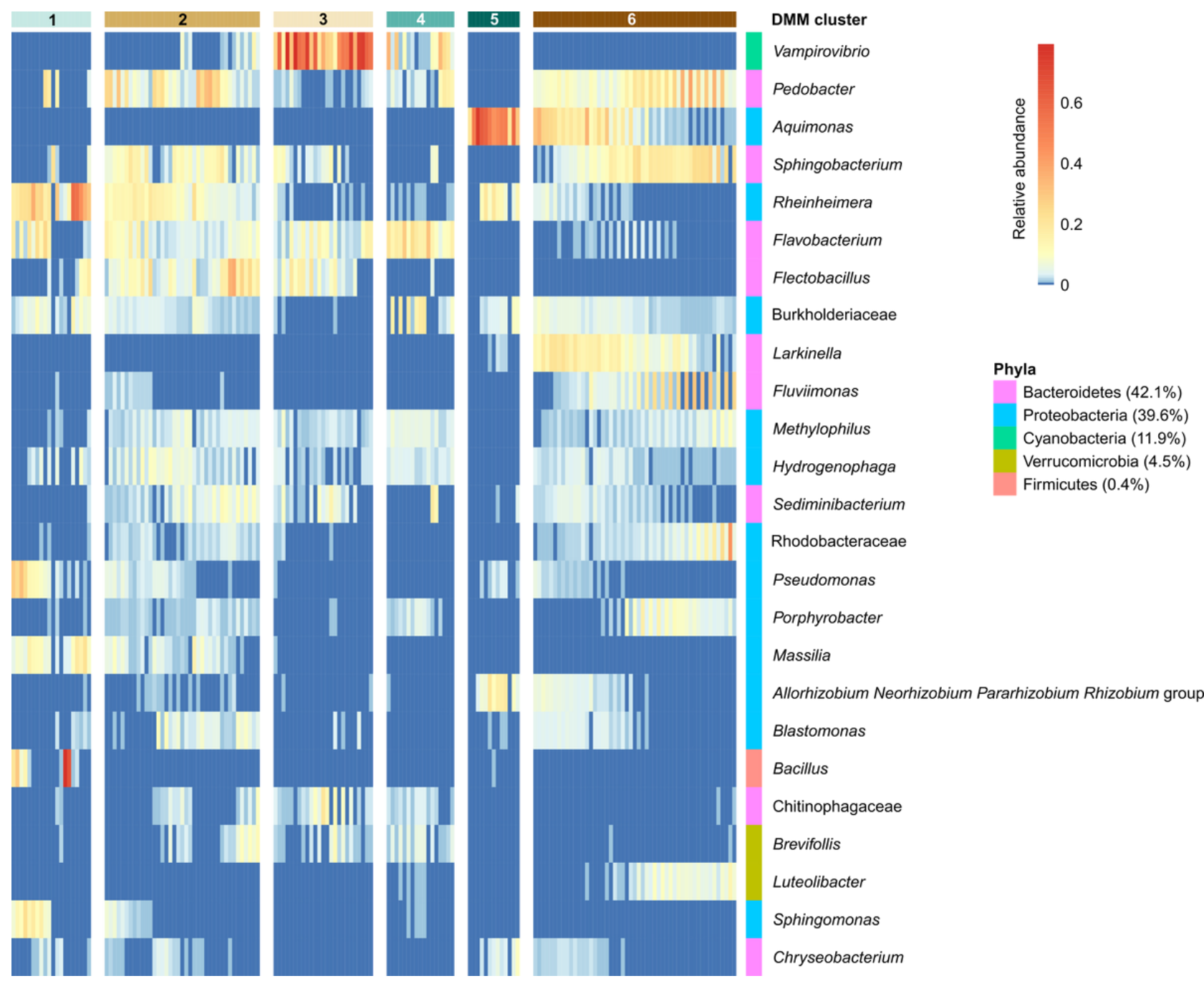

Figure 4

DMM clustering of RAFT baseline experiment 16 S ribosomal RNA gene sequence $(n=165)$. The heatmap shows the relative abundance of the 25 most common genera identified for each sample. Columns represent samples, which are grouped by the respective DMM cluster. Rows are labeled based on genuslevel identification, or by family-level identification when classification was uncertain, with the corresponding phyla annotation indicated by the differentlycolored columns. 


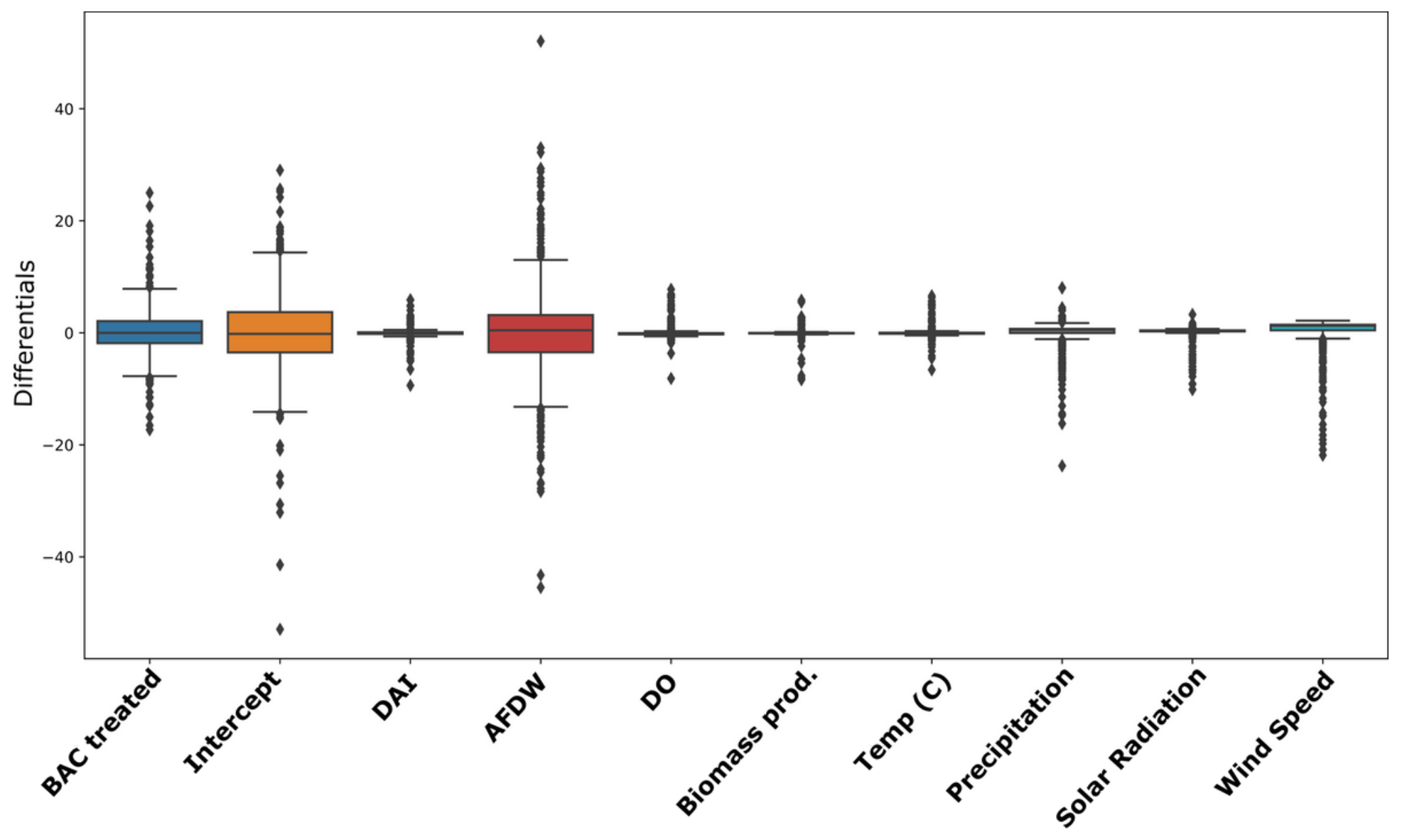

Figure 5

Correlation between sequence variants and variables measured in the outdoor RAFT reactor during cultivation of Chlorella sorokiniana. Boxplots indicate the distribution of differential constants assigned to each $16 \mathrm{~S}$ ribosomal RNA gene sequence variant, based on the multinomial regression against nine variables. Values outside the \pm 1.5 interquartile range were designated as outliers and denoted as points outside the whiskers for each variable.

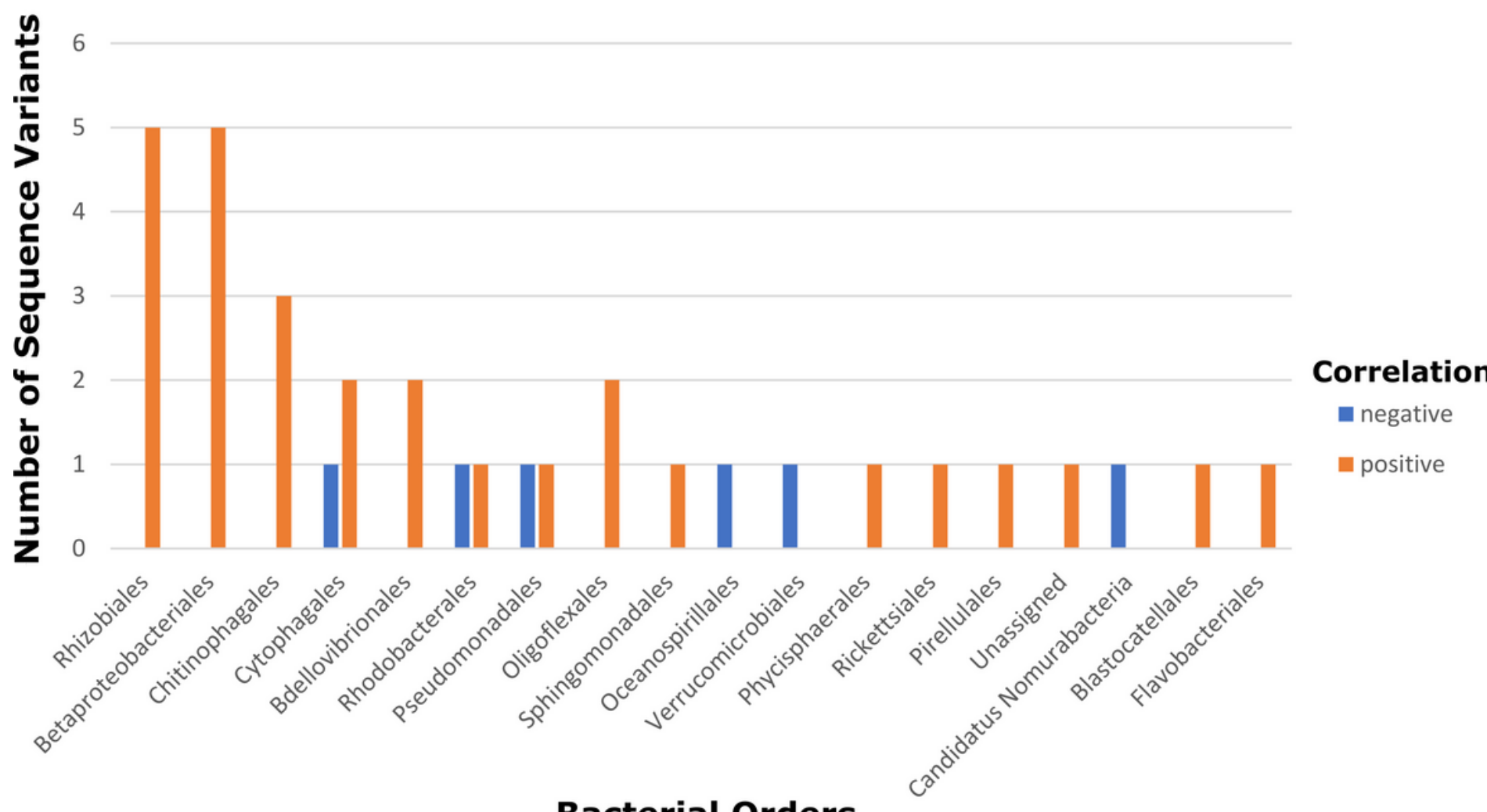


Figure 6

The bacterial orders identified as most highly correlated with biomass productivity of the algal cultures. Counts of sequence variants with multinomial regression differential values for biomass productivity are indicated by a z score of greater than three. The sequence variants are grouped by the assigned taxonomy at the order-level, and bars are color-coded based on the direction of the correlation.

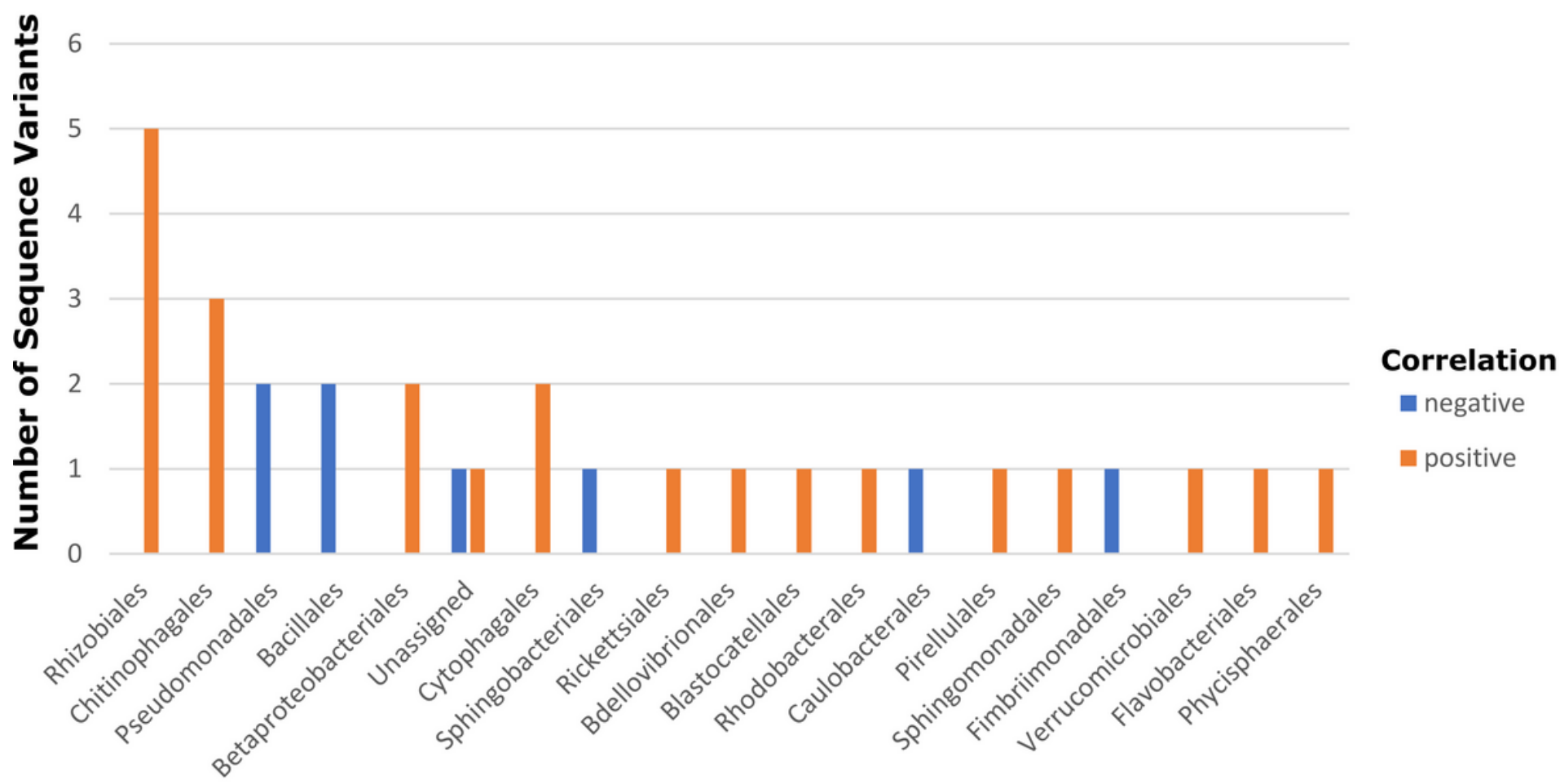

Bacterial Orders

\section{Figure 7}

Bacterial orders most highly correlated with the duration algal cultivation in the outdoor RAFT reactors. Counts of sequence variants with multinomial regression differential values for days after inoculation are indicated by a z score of greater than three. The sequence variants are grouped by their assigned taxonomy at the order-level, and bars are color-coded by the direction of the correlation. 

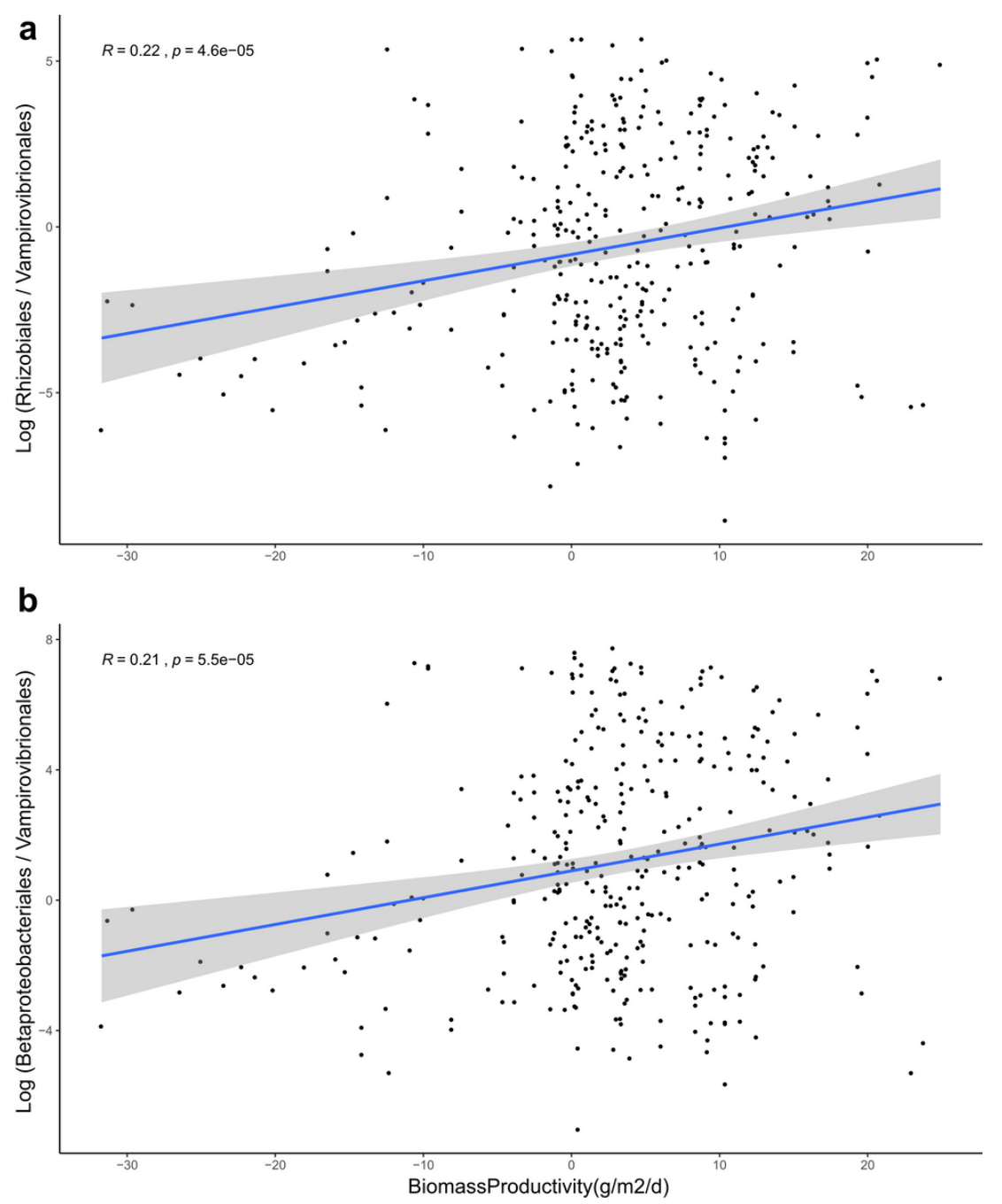

\section{Figure 8}

Log ratio relationship of select bacterial orders to biomass productivity. The log ratios of the relative abundance of a) Betaproteobacteriales ASVs and b) Rhizobiales to Vampirovibrionales for each sample are plotted by their corresponding biomass productivity measure. Lines and grey areas represent linear regression and 95\% confidence intervals, respectively, and $\mathrm{R}$ fitness and $\mathrm{p}$ significance values, based on the Pearson's correlation test. 

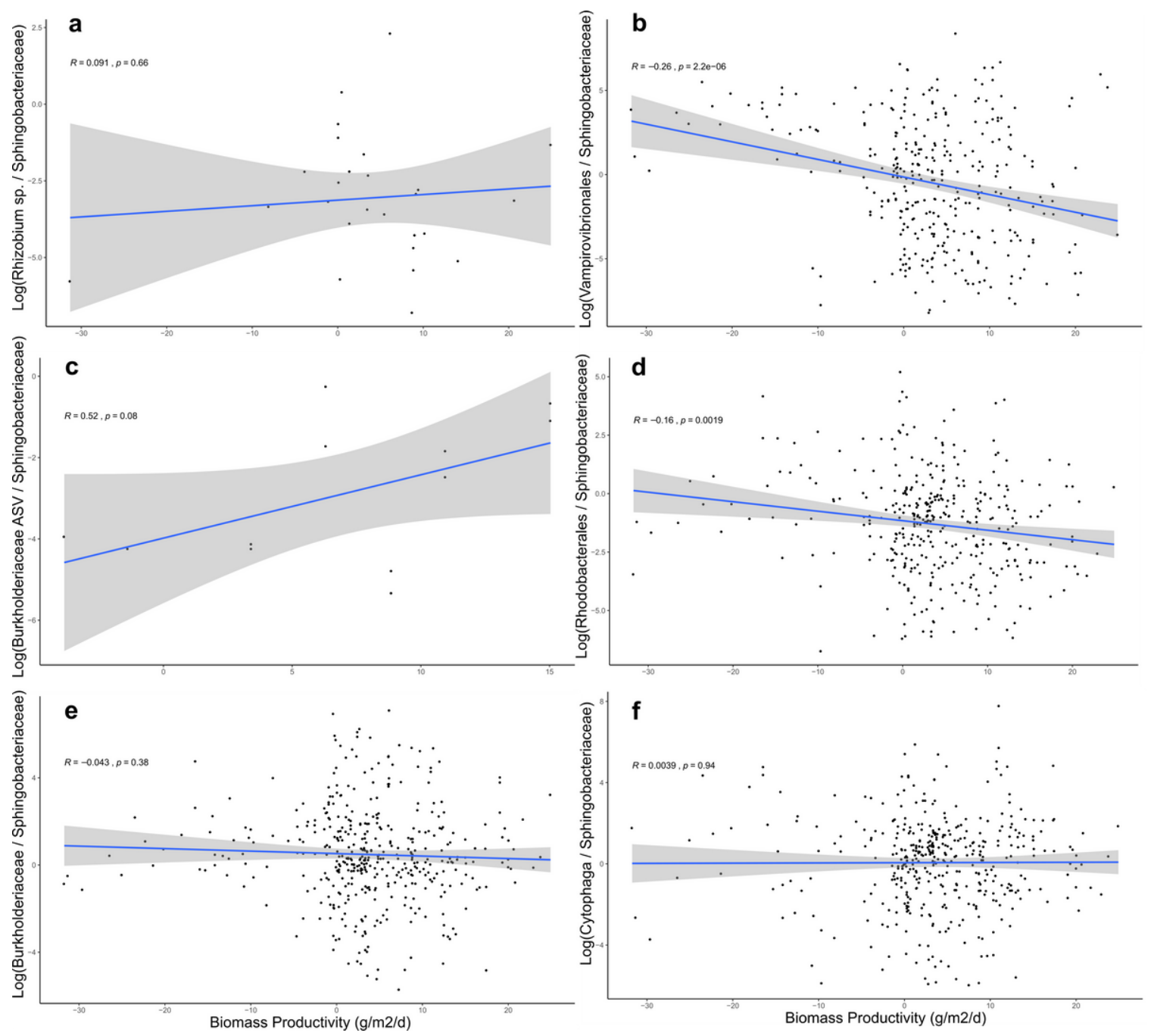

\section{Figure 9}

Log ratio relationships of selected bacterial taxa to algal biomass productivity. The log ratios of the relative abundance of a) Rhizobium sp. (accession no. JX255399), b) Vampirovibrionales, c) Burholderiaceae ASV 21c587e98537024a404d6f041c0fb693, d) Rhodobacterales, e) Burkholderiaceae, and f)

Cytophagales to Sphingobacteriaceae for each sample are plotted by their corresponding biomass productivity measure. Lines and grey areas represent linear regression and $95 \%$ confidence intervals, respectively, and $\mathrm{R}$ fitness and $\mathrm{p}$ significance values, based on the Pearson's correlation test. 
a

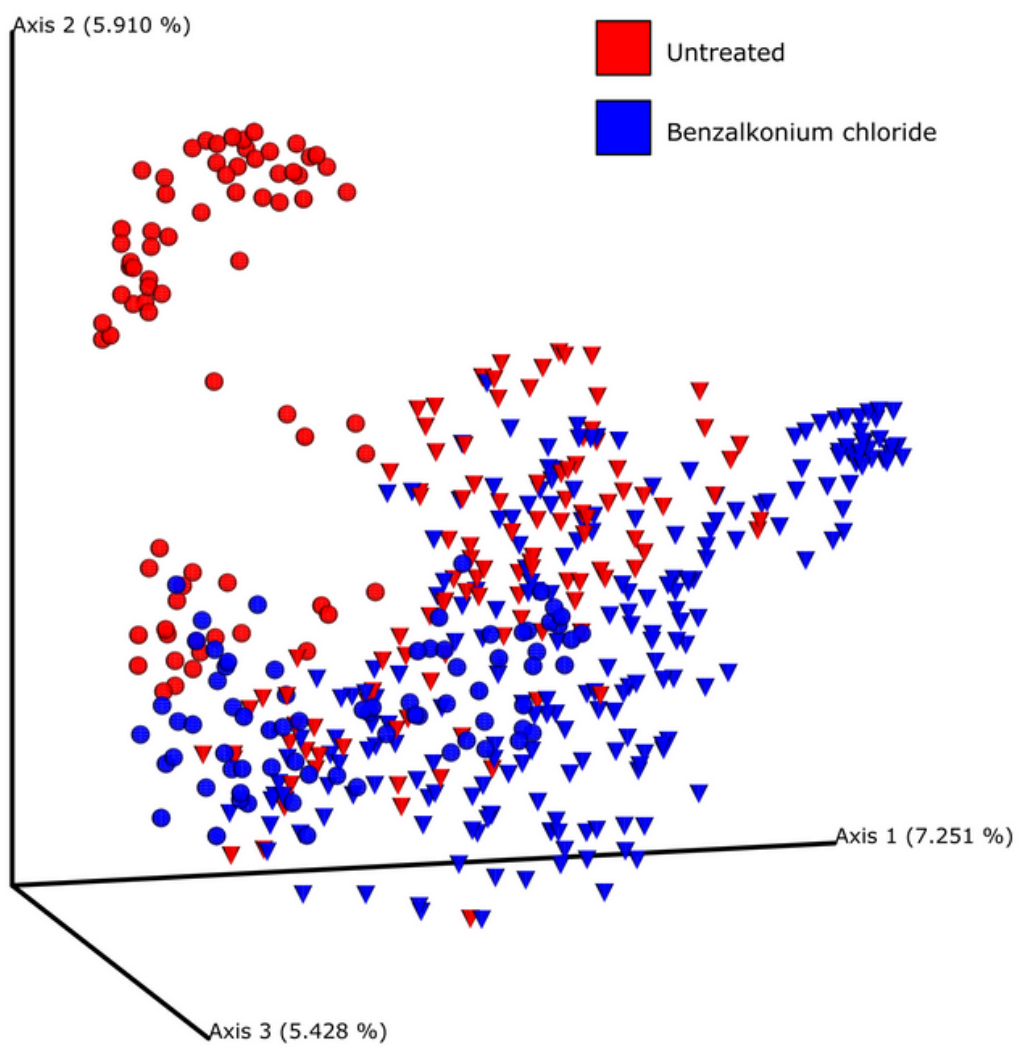

b

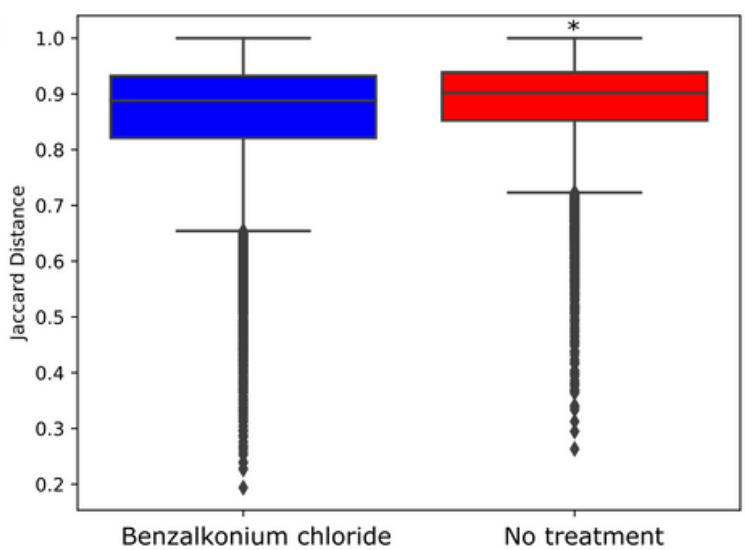

C

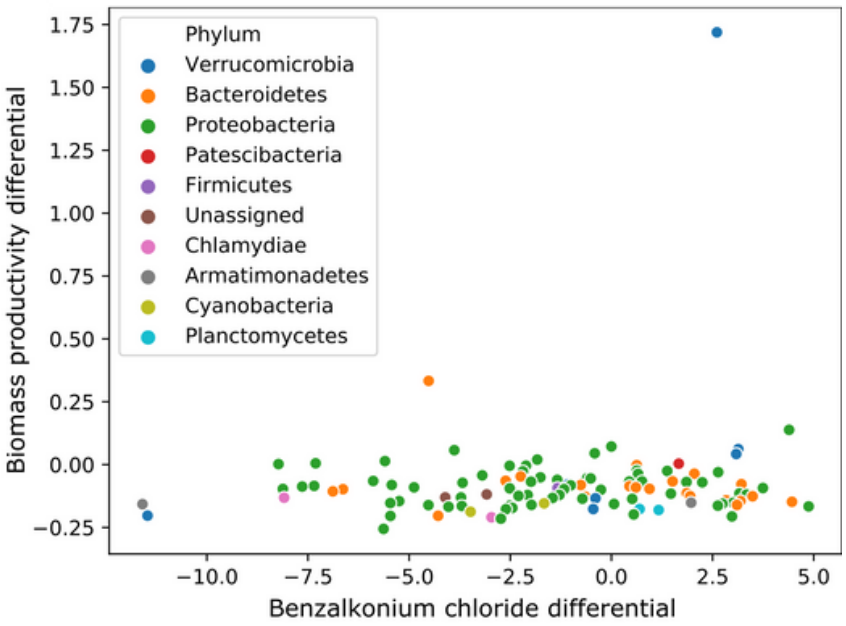

Figure 10

Changes in bacterial community structure associated with benzalkonium chloride treatments. The Jaccard distances between samples collected during RAFT experiments $(n=476)$ are displayed in an a) Principal Coordinate Analysis (PCoA) plot with those collected following benzalkonium chloride treatment colored blue and untreated samples colored red. Samples collected during the baseline experiments are denoted by spheres $(n=148)$ and survey experiments by cones $(n=328)$. Beta diversity comparisons between samples from Benzalkonium chloride treated cultures and untreated are displayed in $b$ ) boxplots as the distance to the centroid of treated samples was determined to be significant by PERMANOVA analysis $(p=0.001)$. The ASVs determined to be significantly differentially abundant between treatments by ANCOM analysis $(n=107)$ are $c)$ plotted according to multinomial model differential values for biomass productivity and BAC treatment variables. 

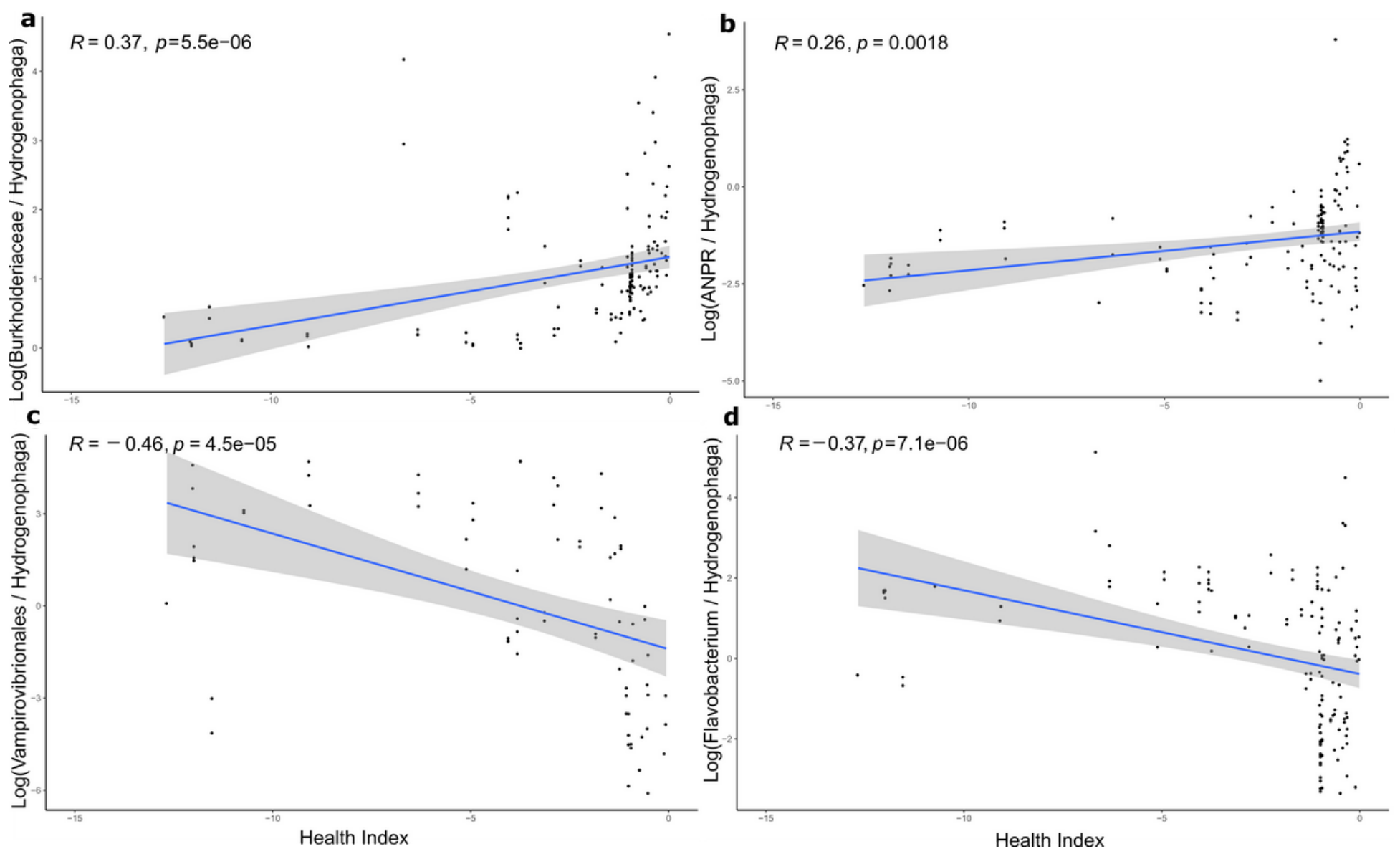

Figure 11

Log ratio relationships of selected bacterial taxa to algal Health Index (HI). The log ratios of the relative abundance of a) Burkholderiaceae, b) AllorhizobiumNeorhizobium-Pararhizobium-Rhizobium (ANPR), c) Vampirovibrionales, d) and Flavobacterium to Hydrogenophaga for each sample are plotted by their corresponding algal $\mathrm{HI}$ measure. Lines and grey areas represent linear regression and $95 \%$ confidence intervals, respectively, and R fitness and p significance values, based on the Pearson's correlation test.

\section{Supplementary Files}

This is a list of supplementary files associated with this preprint. Click to download.

- FigS1baselineminlaplace.pdf

- FigS7comparingvampanprinvHIbioprodafdwratios.png

- FigS3avgDOcomps.png

- FigS6songbirdoutliersHI.png

- Figs4bacbioprodtempboxplots.png

- FigS5invHImodelsimcomparions.png

- FigS2monthydist.png

- tableS1RAFTphycospheremetadata.xIsx

- tableS2differentialstaxa.xlsx 